DRAFT VERSION JUNE 23, 2021

Typeset using LATEX twocolumn style in AASTeX63

\title{
Infrared Properties of Asymptotic Giant Branch Stars in Our Galaxy and the Magellanic Clouds
}

\author{
KYUNG-WON SUH ${ }^{1}$ \\ ${ }^{1}$ Department of Astronomy and Space Science, Chungbuk National University, Cheongju-City, 28644, Republic of Korea
}

(Received October 31, 2019; Revised December 17, 2019; Accepted December 28, 2019)

Submitted to ApJ

\begin{abstract}
We investigate infrared properties of asymptotic giant branch (AGB) stars in our Galaxy and the Magellanic Clouds using various infrared observational data and theoretical models. We use catalogs for the sample of 4996 AGB stars in our Galaxy and about 39,000 AGB stars in the Magellanic Clouds from the available literature. For each object in the sample, we cross-identify the 2MASS, WISE, and Spitzer counterparts. To compare the physical properties of O-rich and C-rich AGB stars in our Galaxy and the Magellanic Clouds, we present IR two color diagrams (2CDs) using various photometric data. We perform radiative transfer model calculations for AGB stars using various possible parameters of central stars and dust shells. Using dust opacity functions of amorphous silicate and amorphous carbon, the theoretical dust shell models can roughly reproduce the observations of AGB stars on various IR 2CDs. Compared with our Galaxy, we find that the Magellanic Clouds are deficient in AGB stars with thick dust shells. Compared with the Large Magellanic Cloud (LMC), the Small Magellanic Cloud (SMC) is more deficient in AGB stars with thick dust shells. This could be because the Magellanic Clouds are more metal poor than our Galaxy and the LMC is more metal rich than the SMC. We also present IR properties of known pulsating variable. Investigating the magnitude distributions at MIR bands for AGB stars in the Magellanic Clouds, we find that the SMC is more deficient in the bright AGB stars at MIR bands compared with the LMC.
\end{abstract}

Keywords: stars: AGB and post-AGB - circumstellar matter - infrared: stars - dust, extinction - radiative transfer

\section{INTRODUCTION}

It is generally believed that asymptotic giant branch (AGB) stars are low to intermediate mass stars $\left(0.5-10 M_{\odot}\right.$ : for solar metallicity; the exact value of the upper limit depends on the treatment of convection: e.g., Siess 2006) in the last evolutionary phases evolving rapidly from the red giant branch into planetary nebulae. Most AGB stars are long-period variables (LPVs) with large amplitude pulsations and they have circumstellar dust envelopes with high mass-loss rates $\left(\dot{M} \sim 10^{-8}-10^{-4} M_{\odot} / y r\right.$; Loup et al. 1993; Suh 2014; Höfner \& Olofsson 2018).

During the thermally pulsing AGB (TP-AGB) phase, the AGB stars show higher mass-loss rates, produce dust grains effectively, and enrich the insterstellar medium in metals and dust. AGB stars are classified as O-rich AGB (OAGB) or C-rich AGB (C-AGB) based on the chemistry of the photosphere and/or the outer dust envelope. Circumstellar dust envelopes around AGB stars produce various IR

Corresponding author: Kyung-Won Suh

kwsuh@chungbuk.ac.kr features. The spectral energy distributions (SEDs) of OAGB stars show $10 \mu \mathrm{m}$ and $18 \mu \mathrm{m}$ features due to amorphous silicate dust. Low mass-loss rate O-AGB (LMOA; $\left.\dot{M} \sim 10^{-8}-10^{-6} M_{\odot} / y r\right)$ stars with thin dust envelopes show the emission features and high mass-loss rate O-AGB (HMOA; $\dot{M} \sim 10^{-5}-10^{-4} M_{\odot} / y r$ ) stars with thick dust envelopes show the absorption features at the same wavelengths (e.g., Suh 1999). The detailed SEDs of LMOA stars can be reproduced by the silicate dust with a mixture of amorphous alumina $\left(\mathrm{Al}_{2} \mathrm{O}_{3}\right.$; Suh 2016) and Fe-Mg oxides (Th. Posch et al. 2001). Featureless amorphous carbon (AMC) dust with a mixture of $\mathrm{SiC}$ and $\mathrm{MgS}$ grains can reproduce the SEDs For C-AGB stars (e.g., Suh 2000; Hony et al. 2002).

During the AGB phase, the abundances of $\mathrm{C}, \mathrm{N}$, and $\mathrm{O}$ in the stellar atmosphere can be changed by the episodic third dredge-up process after each thermal pulse (e.g., Iben \& Renzini 1983). When AGB stars of intermediate mass range (1.55 $M_{\odot} \leq \mathrm{M}<4 M_{\odot}$ : for solar metallicity) go through the carbon dredge-up process and thus the $\mathrm{C} / \mathrm{O}$ ratio is larger than 1, the O-AGB stars may become C-AGB stars (Groenewegen et al. 1995). However, high mass stars (4 $M_{\odot} \leq$ $\mathrm{M}<10 M_{\odot}$ : for solar metallicity) may become hot enough so that hot-bottom burning converts the $\mathrm{C}$ into ${ }^{14} \mathrm{~N}$ by means 
of the CN cycle (Blöcker et al. 2000) and these stars may remain O-AGB, which are typical HMOA stars (or OH/IR stars) with thick dust envelopes and high mass-loss rates.

Various IR observational data at NIR, MIR, and FIR bands are available from the Infrared Astronomical Satellite (IRAS), Infrared Space Observatory (ISO), Midcourse Space Experiment (MSX), AKARI, Two-Micron All-Sky Survey (2MASS), Wide-field Infrared Survey Explorer (WISE), and Spitzer. These data have been very useful to identify new AGB stars and understand the nature of them.

A catalog of AGB stars for 3003 O-AGB and 1168 C-AGB objects in our Galaxy was presented by Suh \& Kwon (2011). Suh \& Hong (2017) presented a revised list of 3828 O-AGB and 1168 C-AGB stars. IR two-color diagrams (2CDs) have been useful to study the properties of central stars and dust envelopes for a large sample of AGB stars (e.g., Suh \& Kwon 2011; Suh 2015). Suh (2018) presented various IR 2CDs using the IRAS, 2MASS, AKARI, and WISE data for the AGB stars in our Galaxy.

Thanks to the optical gravitational lensing experiment (OGLE) projects (Soszyński et al. 2009) and Spitzer Space Telescope Legacy program 'Surveying the Agents of a Galaxy Evolution' (SAGE; Meixner et al. 2006), a much larger number of AGB stars in the Large Magellanic Cloud (LMC) and Small Magellanic Cloud (SMC) are identified and studied. Using the Infrared Spectrograph (IRS) data on the Spitzer Space Telescope, Jones et al. (2014) found that amorphous silicate dust grains with contributions from amorphous alumina and metallic iron, which is similar to the grain mixture for LMOA stars in our Galaxy, provides a good fit to the observed spectra for a number of O-AGB stars in the LMC. Sloan et al. (2016) found that AMC dust with mixture of $\mathrm{SiC}$ and $\mathrm{MgS}$ grains fit C-AGB stars in the LMC and SMC.

Ventura et al. (2016) studied infrared colors of C-AGB stars in the Magellanic Clouds and found the redder infrared colors of C-AGB stars in the LMC compared to their counterparts in the SMC. Groenewegen \& Sloan (2018) investigated mass loss and luminosity in a sample of AGB stars in our Galaxy, the Magellanic Clouds, and other nearby galaxies. Nanni et al. (2019) investigated the mass-loss and dust production rates of C-AGB stars in the Magellanic Clouds and found a tail of extreme mass-losing C-AGB stars in the LMC with low gas-to-dust ratios that is not present in the SMC.

In this work, we investigate IR properties of AGB stars in our Galaxy and the Magellanic Clouds for a large sample of the objects and compare them with theoretical models. We present various IR 2CDs for the large sample of AGB stars using the 2MASS, WISE, and Spitzer data. We use theoretical dust shell models for AGB stars and compare the theory with the observations. We present infrared properties of known pulsating variables in our Galaxy and the Magellanic Clouds. For AGB stars in the Magellanic Clouds, we investigate magnitude distributions at MIR bands. And we compare the IR properties of AGB stars in our Galaxy and the Magellanic Clouds.

\section{SAMPLE STARS}

We use catalogs of AGB stars in our Galaxy and the Magellanic Clouds from the available literature. Table 1 lists the reference, total number of objects, and numbers of the crossidentified 2MASS, WISE, and Spitzer counterparts for each class.

\subsection{Infrared Photometric Data}

IRAS and AKARI data have been very useful for studying AGB stars in our Galaxy (e.g., Suh \& Kwon 2011; Suh 2018). Though they were also useful for studying AGB stars in the Magellanic Clouds (e.g., Jones et al. 2014), the number of the cross-identified objects was very limited because of the relatively large beam sizes and weak sensitivities.

2MASS (Cutri et al. 2003) provided fluxes at $\mathbf{J}(1.25$ $\mu \mathrm{m}), \mathrm{H}(1.65 \mu \mathrm{m})$, and $\mathrm{K}(2.16 \mu \mathrm{m})$ bands. The field of view (FOV) pixel size of the 2MASS image is $2^{\prime \prime}$. The WISE (Wright et al. 2010) mapped the sky at 3.4, 4.6, 12 , and $22 \mu \mathrm{m}$. For the four WISE bands (W1, W2, $\mathrm{W} 3$, and W4), the FOV pixel sizes are 2 '.75, 2".75, 2..75, and $5^{\prime \prime} .5$, and the $5 \sigma$ photometric sensitivities are 0.068 , $0.098,0.86$, and $5.4 \mathrm{mJy}$ (http://wise2.ipac.caltech.edu/docs/ release/allsky/expsup/sec1_1.html). The WISE data have been useful for studying AGB stars in our Galaxy (e.g., Suh 2018) and they would be also useful for studying AGB stars in the Magellanic Clouds.

The Spitzer Space Telescope (Gehrz et al. 2007) had infrared array camera (IRAC; 3.6, 4.5, 5.8, and $8.0 \mu \mathrm{m}$ ) and multiband imaging photometer (MIPS; 24, 70, and $160 \mu \mathrm{m}$ ) bands. For the four IRAC bands, and the $5 \sigma$ photometric sensitivities are 1.3, 2.7, 18, and $22 \mu \mathrm{Jy}$ with the FOV pixel size of 1 !"2. For the MIPS band at $24 \mu \mathrm{m}$, the $5 \sigma$ photometric sensitivity is $110 \mu \mathrm{Jy}$ and the FOV pixel size is 2 .' 5 .

Table 2 lists the IR bands used in this work. For each band, the reference wavelength $\left(\lambda_{r e f}\right)$ and zero magnitude flux (ZMF) value, which are useful to to obtain theoretical models colors (see Section 4.2), are also shown.

In this work, we use only good quality observational data at all wavelength bands for the 2MASS and WISE photometric data (quality A for the 2MASS; quality A or B for the WISE). For the Spitzer photometric data, we use all of the available data because we use the SAGE catalogs with high reliability, which were extracted from the full list by placing strict restrictions on the source quality (see Sections 2.2 and 2.3).

\subsection{AGB stars in our Galaxy}

A catalog of AGB stars for 3003 O-AGB and 1168 C-AGB objects in our Galaxy was presented by Suh \& Kwon (2011). Suh \& Hong (2017) presented a revised list of 3828 O-AGB and $1168 \mathrm{C}$-AGB stars based on the IRAS point source catalog (PSC). The classification was based on IR and optical spectroscopy, IR photometry, and maser observations (see Suh \& Kwon 2011; Suh \& Hong 2017). The sample of 4996 Galactic AGB stars is composed of Mira variables (O-AGB: 1444; C-AGB: 292), semiregular variables (SRVs; O-AGB: 
Table 1. Sample of AGB stars in our Galaxy and the Magellanic Clouds

\begin{tabular}{|c|c|c|c|c|c|c|}
\hline Class & Reference & Total Number & 2MASS & WISE & IRAC $^{1}$ & MIPS $^{1}$ \\
\hline O-AGB (our Galaxy) & Suh \& Hong (2017) & 3828 & 3828 & 3822 & $591(192)$ & $784(303)$ \\
\hline C-AGB (our Galaxy) & Suh \& Hong (2017) & 1168 & 1168 & 1167 & $51(7)$ & $67(18)$ \\
\hline O-AGB (LMC-OGLE3) & Soszyński et al. (2009) & 37,203 & 37,194 & 34,871 & 36,113 & 2540 \\
\hline C-AGB (LMC-OGLE3) & Soszyński et al. (2009) & 9264 & 9257 & 9092 & 8970 & 5238 \\
\hline O-AGB (SMC-OGLE3) & Soszyński et al. (2011) & 2511 & 2511 & 2438 & 2477 & 164 \\
\hline C-AGB (SMC-OGLE3) & Soszyński et al. (2011) & 2761 & 2757 & 2724 & 2743 & 1173 \\
\hline O-AGB (LMC-SAGE) & Riebel et al. (2012) & $26,231^{2}$ & 26,231 & 25,745 & 26,080 & 7570 \\
\hline C-AGB (LMC-SAGE) & Riebel et al. (2012) & $7306^{3}$ & 7276 & 7268 & 7256 & 6743 \\
\hline O-AGB (SMC-SAGE) & Srinivasan et al. (2016) & 3624 & 3624 & 3538 & 3624 & 160 \\
\hline C-AGB (SMC-SAGE) & Srinivasan et al. (2016) & 2118 & 2117 & 2101 & 2118 & 1134 \\
\hline O-AGB (LMC-SAGE-S) ${ }^{4}$ & Jones et al. (2017) & 77 & 77 & 75 & 74 & 75 \\
\hline C-AGB (LMC-SAGE-S) ${ }^{4}$ & Sloan et al. (2016) & $151^{5}$ & 135 & 145 & 145 & 143 \\
\hline O-AGB (SMC-SAGE-S) ${ }^{4}$ & Kraemer et al. (2017) & 5 & 5 & 5 & 5 & 5 \\
\hline C-AGB (SMC-SAGE-S) ${ }^{4}$ & Sloan et al. (2016) & 40 & 40 & 38 & 40 & 37 \\
\hline
\end{tabular}

${ }^{1}$ the number in parenthesis for the objects in our Galaxy denotes the number of the data with small deviations in the S5[24] flux (see Section 2.2). ${ }^{2} 21$ newly identified SAGE O-AGB objects from Jones et al. (2017) are added. ${ }^{3} 13$ newly identified SAGE C-AGB objects from Jones et al. (2017) are added. ${ }^{4}$ Identified from the SAGE IRS spectroscopy. ${ }^{5} 7$ newly identified SAGE-S C-AGB objects from Jones et al. (2017) are added.

Table 2. IR bands and zero magnitude flux values

\begin{tabular}{lllll}
\hline \hline Band & $\lambda_{r e f}(\mu \mathrm{m})$ & ZMF $(\mathrm{Jy})$ & Remark & Reference $^{1}$ \\
\hline $\mathrm{J}[1.2]$ & 1.235 & 1594 & 2MASS & Cohen et al. (2003) \\
$\mathrm{H}[1.7]$ & 1.662 & 1024 & 2MASS & Cohen et al. (2003) \\
K[2.2] & 2.159 & 666.7 & 2MASS & Cohen et al. (2003) \\
W1[3.4] & 3.35 & 306.682 & WISE & Jarrett et al. (2011) \\
S1[3.6] & 3.55 & 280.9 & Spitzer & A \\
S2[4.5] & 4.493 & 179.7 & Spitzer & A \\
W2[4.6] & 4.60 & 170.663 & WISE & Jarrett et al. (2011) \\
S3[5.8] & 5.731 & 115.0 & Spitzer & A \\
S4[8.0] & 7.872 & 64.9 & Spitzer & A \\
W3[12] & $12.0(11.56)$ & $28.3(29.045)$ & WISE & Jarrett et al. (2011) \\
W4[22] & 22.08 & 8.284 & WISE & Jarrett et al. (2011) \\
S5[24] & 23.68 & 7.17 & Spitzer & B \\
\hline
\end{tabular}

${ }^{1}$ A: https://irsa.ipac.caltech.edu/data/SPITZER/docs/irac/iracinstrumenthandbook, B: https://irsa.ipac.caltech.edu/data/SPITZER/docs/mips/mipsinstrumenthandbook. ${ }^{2}$ For W3[12], we use a new reference wavelength and zero magnitude flux for theoretical models (original values are given in parenthesis; see 4.2).

167; C-AGB: 178), and other types according to the American association of variable star observers (AAVSO) international variable star index (VSX; Watson et al. 2019). Among the 3828 O-AGB stars, 1520 objects are known to be OH/IR stars (see Section 2.5), from which 271 objects are known to be Miras according to the AAVSO. Note that most of the Galactic AGB stars with thick dust envelopes are not listed in the AAVSO catalog, which is mainly based on optical observations.

Because IRAS has a large beam size, it is tricky to find appropriate 2MASS, WISE, or Spitzer counterparts using the
IRAS PSC position (see Suh 2018). We find the AKARI PSC, 2MASS, and WISE counterparts as described in Suh (2018), which considered the beam sizes and compared the fluxes. To find the Spitzer IRAC and MIPS counterparts, we use the same method that was used for finding the WISE counterpart (Suh 2018). We make cross identification to the Spitzer point sources by using the 'A 24 and 70 Micron Survey of the Inner Galactic Disk with MIPS' (MIPSGAL) cata$\log$, which provides the Spitzer photometric data for 933,818 sources in our Galaxy. Table 1 lists the sample AGB stars in our Galaxy and numbers of the cross-matched 2MASS, WISE, and Spitzer counterparts.

The upper panel of Figure 1 shows the comparison of the IRAS [25] $(25 \mu \mathrm{m})$ flux with the Spitzer S5[24] $(24 \mu \mathrm{m})$ flux for AGB stars in our Galaxy. For the Spitzer counterparts, the Spitzer fluxes drop abnormally compared with other measurements at nearby wavelengths. This would be mainly because of the saturation effect of the Spitzer data for the bright Galactic AGB stars. There is a similar effect for the WISE data for AGB stars in our Galaxy, but it is known to be minor for a considerable portion of them (see Suh 2018). When we remove the objects with very large decreases in Spitzer S5[24] flux from the IRAS [25] flux (more than 2.5 mag; see Figure 1), more reliable data points with small deviations (see Table 1) can be distinguishable. The lower panel of Figure 1 shows the comparison of the WISE W2[4.6] flux with the Spitzer S3[5.8] flux for AGB stars in our Galaxy, which shows the saturation effect only for bright objects at W2[4.6]. We also show the objects with with small deviations in the Spitzer S5[24] flux. 

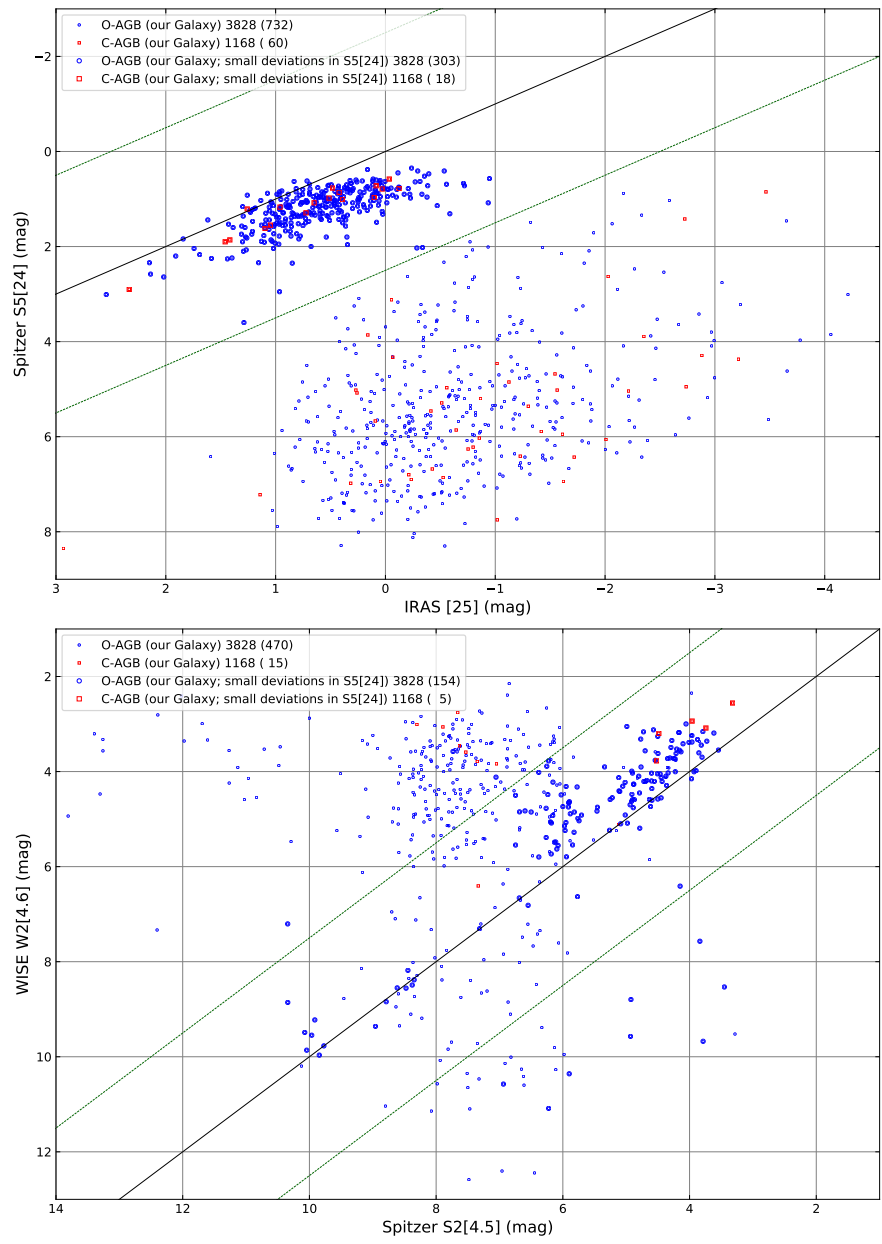

Figure 1. Comparison of the fluxes (in mag) at Spitzer and other bands (Spitzer IRAC S5[24] versus IRAS PSC [25]; Spitzer IRAC S3[5.8] versus WISE W2[4.6]) for AGB stars in our Galaxy (see Table 1). For each class, the number of objects is shown. The number in parenthesis denotes the number of the plotted objects with good quality observed data. See Section 2.2.

\subsection{AGB stars in the Magellanic Clouds}

The optical gravitational lensing experiment (OGLE) projects detected many LPVs in the Magellanic Clouds. The fourth part of the OGLE-III Catalog of Variable Stars presented 91,995 long-period variables (LPVs) in the LMC (Soszyński et al. 2009). The sample is composed of 1663 Mira variables, 11,132 SRVs, and 79,200 small amplitude red giants (OSARGs). We use the 46,467 AGB candidate objects (1663 Miras, 11,132 SRVs, and 33,672 bright OSARGs) as the sample AGB stars in the LMC-OGLE3 catalog. There are 37,203 O-AGB and 9264 C-AGB objects, which are classified based on their color selection method using the photometric data at optical and NIR bands, in the LMC-OGLE3 sample.

The thirteenth part of the OGLE-III Catalog of Variable Stars (OIII-CVS) contains 19,384 LPVs detected in the SMC (Soszyński et al. 2011). They are composed of 352 Miras,
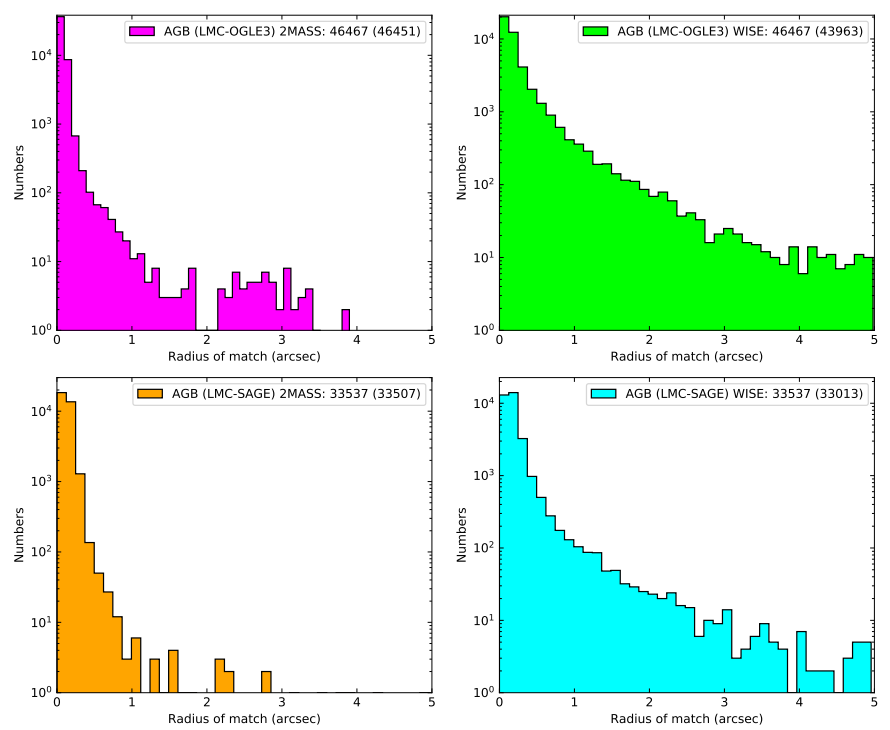

Figure 2. Number distributions of cross-match angular distances for OGLE3 and SAGE AGB stars in the LMC (see Table 1) to the 2MASS and WISE point sources. For each sample, the number of objects is shown. The number in parenthesis denotes the number of the cross matches. See Section 2.4.

2222 SRVs, and 16,810 OSARGs. We use the 5272 AGB candidate objects (352 Miras, 2222 SRVs, and 2698 bright OSARGs) as the sample AGB stars in the SMC-OGLE3 catalog. There are 2511 O-AGB and 2761 C-AGB objects, which are classified based on their color selection method using the photometric data at optical and NIR bands, in the SMC-OGLE3 sample.

The LMC and SMC were imaged as a part of the SAGE program (Meixner et al. 2006). The SAGE program has provided a complete infrared survey of the evolved star population in the LMC and SMC. The Spitzer Infrared Spectrograph (IRS; $\lambda=5.2-38 \mu \mathrm{m}$ ) has taken high resolution spectra for many AGB stars in the LMC and SMC.

Analyzing the Spitzer data of the SAGE program, Riebel et al. (2012) presented a list 33,503 candidate objects for AGB stars in the LMC. They classified them into 26,210 OAGB and 7293 C-AGB objects based on the comparison of the photometric data at NIR and MIR bands with their Grid of AGB and RSG ModelS (GRAMS). By analyzing the IRS spectral data in the SAGE program, Jones et al. (2017) identified and classified many AGB stars, from which 34 objects were new AGB stars compared with the list of Riebel et al. (2012). Therefore, the total number of sample AGB stars in the LMC is 33,537 (O-AGB: 26,231; C-AGB:7306).

Analyzing the data of the SAGE program for the SMC, Srinivasan et al. (2016) presented a list of 9,621 candidate objects for evolved stars. Based on the selection criteria presented by Boyer et al. (2011), they classified them into 2485 O-AGB, 1714 C-AGB, 1198 anomalous-AGB, 341 extremeAGB, and other objects. When we compare the list with the new classification by Kraemer et al. (2017), which presented a list of evolved stars in the SMC by analyzing the Spitzer 

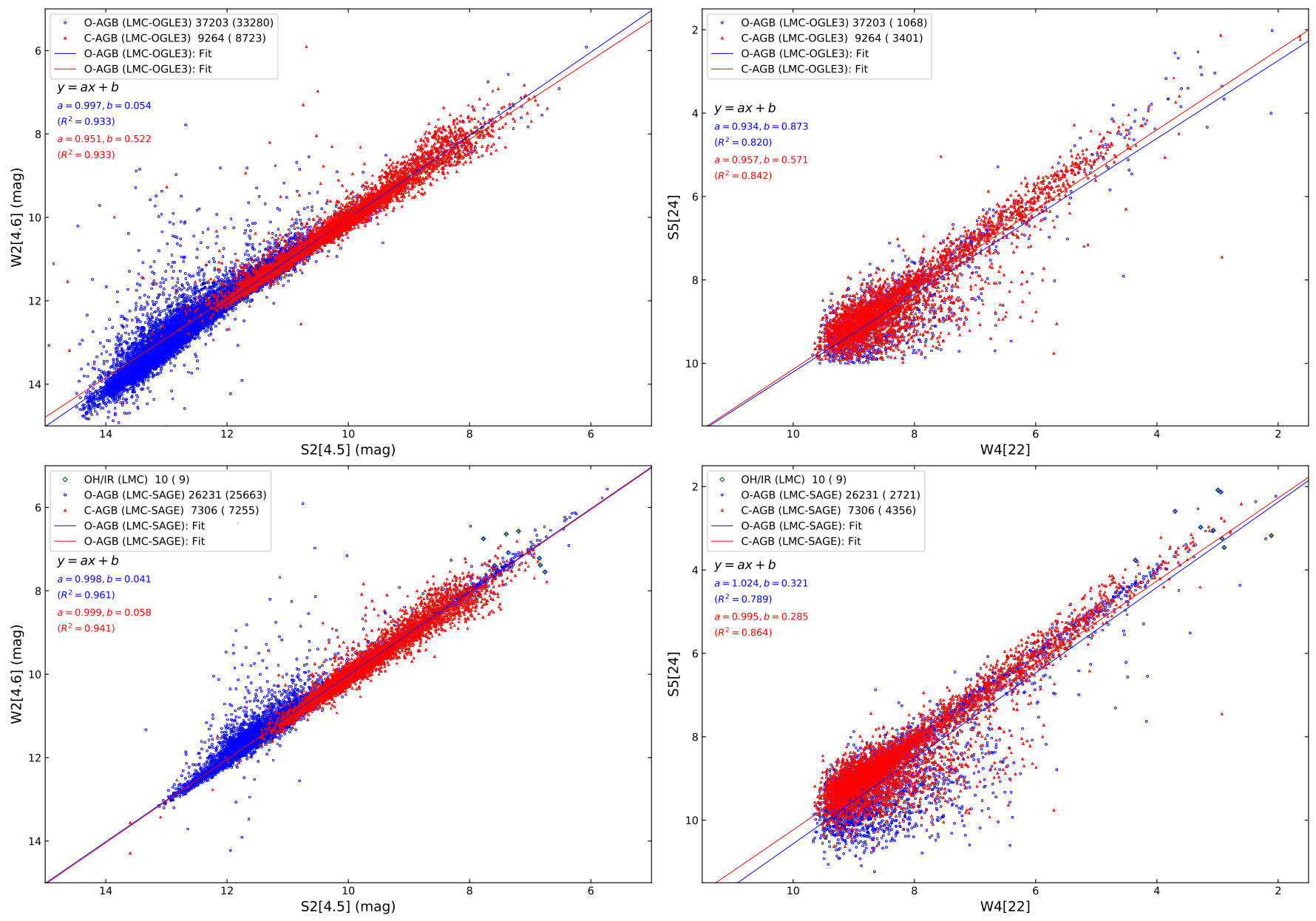

Figure 3. Comparison of the fluxes (in mag) at Spitzer and WISE bands for cross-identified objects in the LMC (OGLE3 and SAGE) sample stars. For each sample, the number of objects is shown. The number in parenthesis denotes the number of the plotted objects with good quality observed data. The coefficients of determination $\left(R^{2}\right)$ for the linear relations are also shown. See Section 2.4.

IRS spectral data, we find that four object in the list of Srinivasan et al. (2016) are newly classified as AGB stars. When we select the AGB stars in the list, we have 5742 AGB stars in the SMC that are classified as 3624 O-AGB and $2118 \mathrm{C}$ AGB based on the GRAMS chemical classification.

Sloan et al. (2016) presented a list 184 C-AGB stars (LMC:144; SMC: 40) by analyzing the SAGE IRS data. When we combine the lists from Sloan et al. (2016), Jones et al. (2017), and Kraemer et al. (2017), there are 77 O-AGB and $151 \mathrm{C}$-AGB stars in the LMC and 5 O-AGB and $40 \mathrm{C}$ AGB stars in the SMC, which are identified from the SAGE IRS data. These SAGE IRS (SAGE-S) sample stars that are identified by the IRS spectra would be more reliable sample of AGB stars in the LMC and SMC.

Table 1 lists the sample AGB stars in the LMC and SMC. Note that all of the objects in the LMC-SAGE-S and SMCSAGE-S samples are already included in the LMC-SAGE and SMC-SAGE samples.

\subsection{AGB stars in the Magellanic Clouds - Cross matches}

For SAGE sample objects in the LMC and SMC, we make cross identification to the sources in the OGLE3 catalogs by finding the nearest sources within $5^{\prime \prime}$. From the 33,537 LMCSAGE AGB sample objects, 22,327 objects (67\%) are duplicated with the LMC-OGLE3 sample (1522 Miras, 9534 SRVs, and 11,271 OSARGs). Though the chemical classification methods for the two samples (OGLE3 and SAGE) are different (see Section 2.3), 19,082 objects (85\%) from the duplicated 22,327 objects are classified as the same class (O-AGB or C-AGB). From the 5742 SMC-SAGE AGB sample objects, 4837 objects $(84 \%)$ are duplicated with the SMC-OGLE3 sample (341 Miras, 2036 SRVs, and 2460 OSARGs).

For all OGLE3 and SAGE sample objects in the LMC and SMC, we make cross identification to the sources in the 2MASS and WISE PSC catalogs by finding the nearest sources within $5^{\prime \prime}$. For WISE data, multiple sample objects may have the same cross-matched WISE point source. So we have checked all of the duplicated cross-matches and selected only one nearest sample object for the one WISE point source. Figure 2 shows number distributions of the crossmatch angular distances for OGLE3 and SAGE sample objects in the LMC to the 2MASS and WISE point sources. 

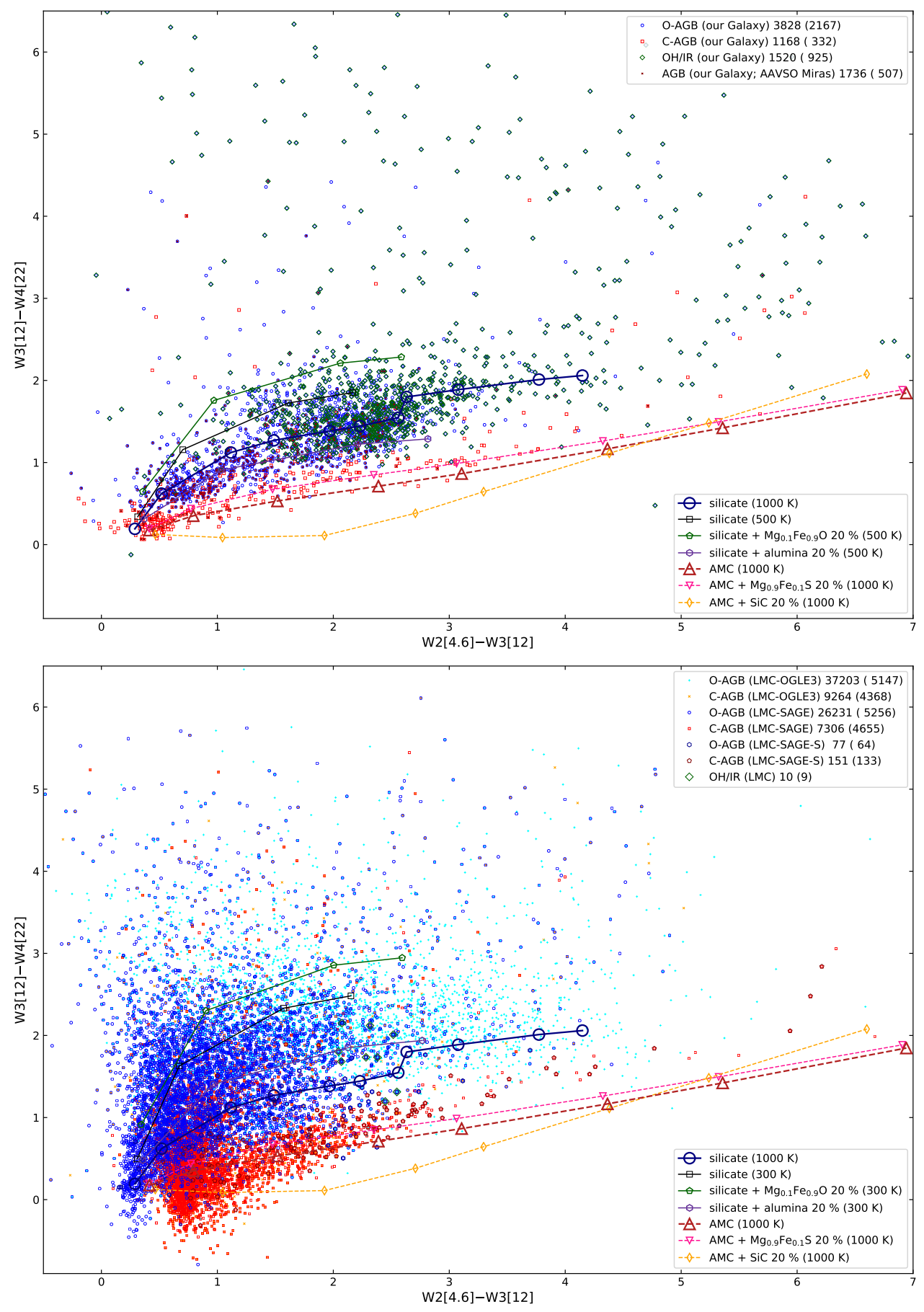

Figure 4. WISE 2CDs for AGB stars in our Galaxy and the LMC (OGLE3 and SAGE samples) compared with theoretical models (see Section 4). For O-AGB models (Silicate $T_{c}=1000 \mathrm{~K}$ ): $\tau_{10}=0.001,0.01,0.05,0.1,0.5,1,3,7,15,30$, and 40 from left to right. For C-AGB models (AMC $T_{c}=1000 \mathrm{~K}$ ): $\tau_{10}=0.001,0.01,0.1,0.5,1,2,3$, and 5 from left to right. For each class, the number of objects is shown. The number in parenthesis denotes the number of the plotted objects on the $2 \mathrm{CD}$ with good quality observed colors. 

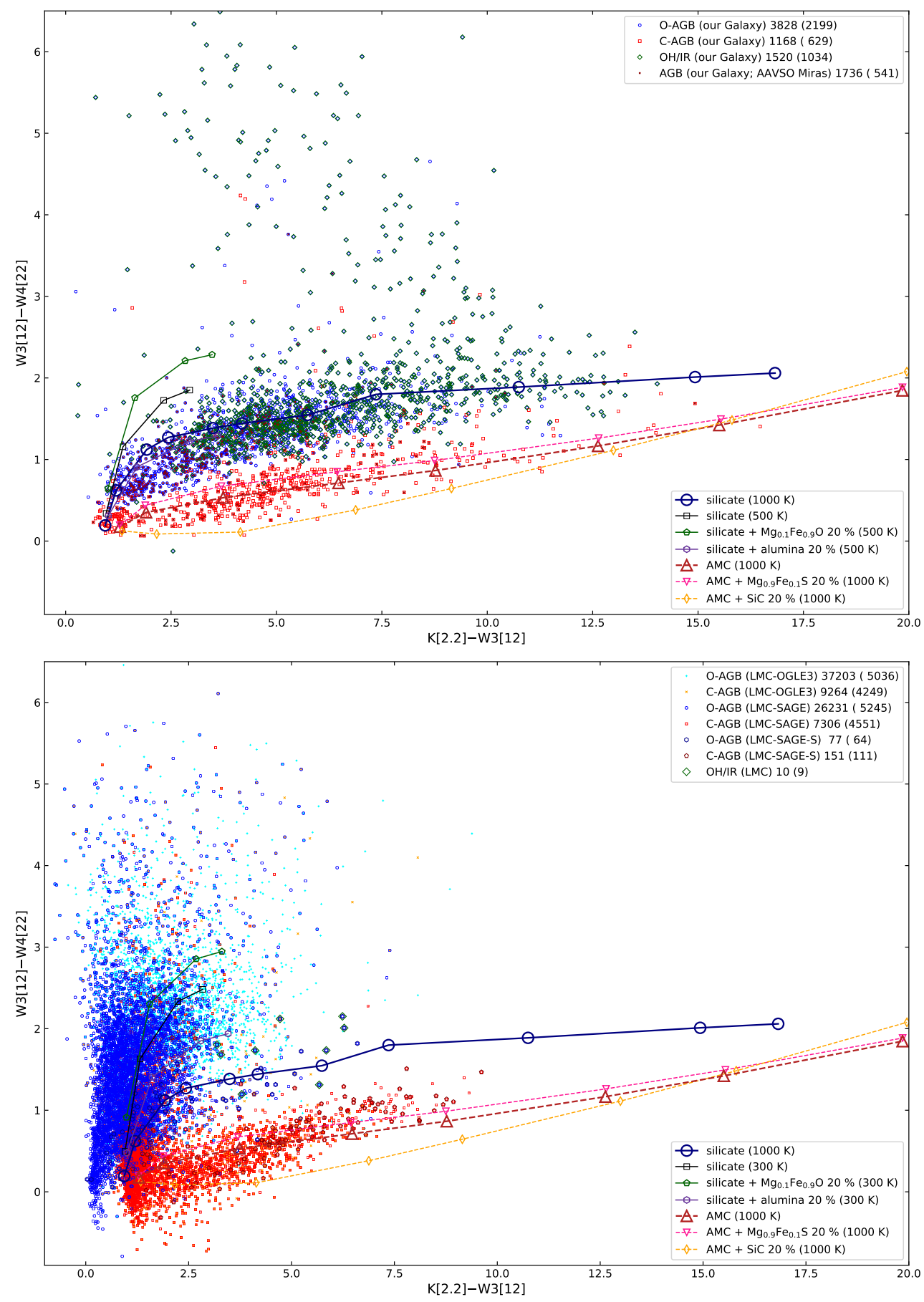

Figure 5. WISE-2MASS 2CDs for AGB stars in our Galaxy and the LMC (OGLE3 and SAGE samples) compared with theoretical models (see Section 4). For O-AGB models (Silicate $T_{c}=1000 \mathrm{~K}$ ): $\tau_{10}=0.001,0.01,0.05,0.1,0.5,1,3,7,15,30$, and 40 from left to right. For C-AGB models (AMC $T_{c}=1000 \mathrm{~K}$ ): $\tau_{10}=0.001,0.01,0.1,0.5,1,2,3$, and 5 from left to right. For each class, the number of objects is shown. The number in parenthesis denotes the number of the plotted objects on the $2 \mathrm{CD}$ with good quality observed colors. 

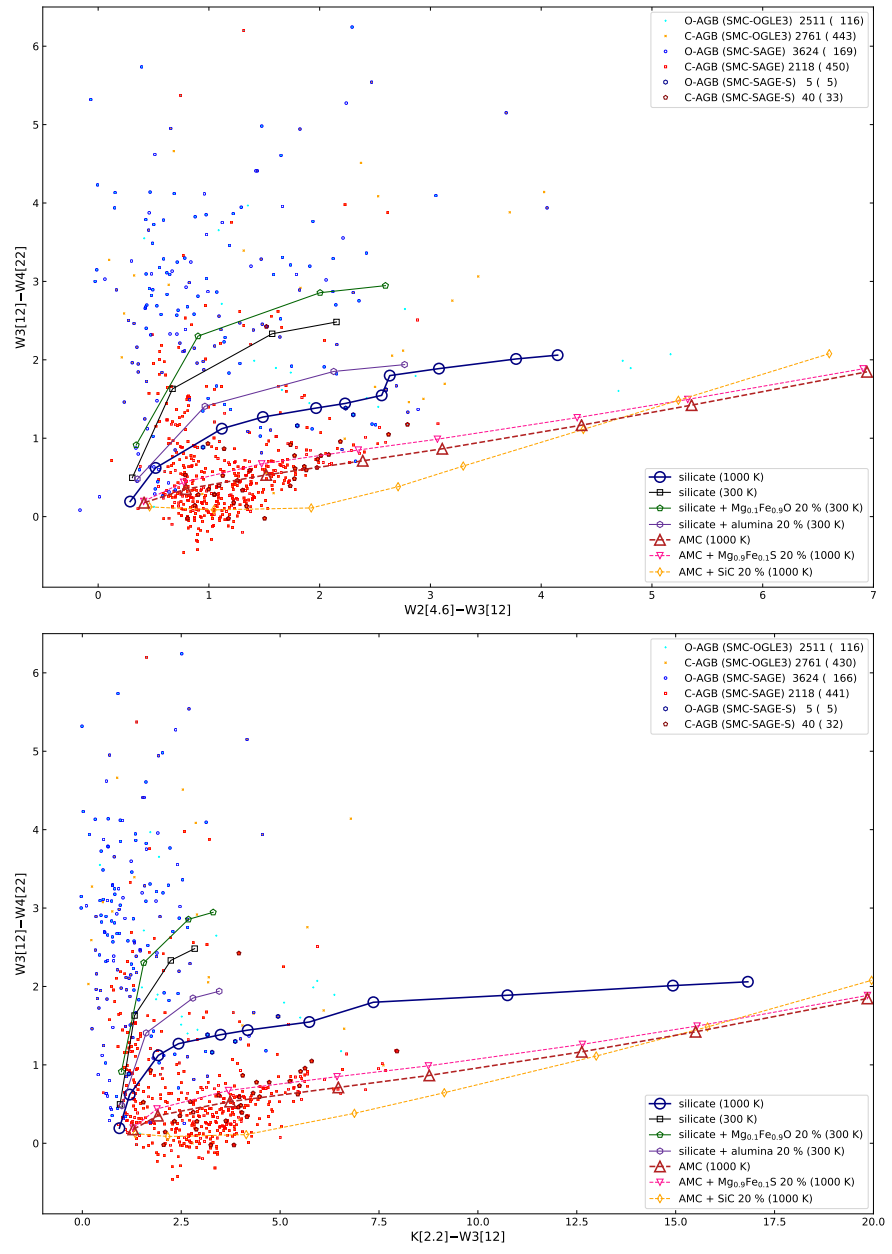

Figure 6. WISE-2MASS 2CDs for AGB stars in the SMC (OGLE3 and SAGE samples) compared with theoretical models (see Section 4). For O-AGB models (Silicate $T_{c}=1000 \mathrm{~K}$ ): $\tau_{10}=0.001$, $0.01,0.05,0.1,0.5,1,3,7,15,30$, and 40 from left to right. For C-AGB models $\left(\right.$ AMC $\left.T_{c}=1000 \mathrm{~K}\right): \tau_{10}=0.001,0.01,0.1,0.5$, $1,2,3$, and 5 from left to right. For each class, the number of objects is shown. The number in parenthesis denotes the number of the plotted objects on the $2 \mathrm{CD}$ with good quality observed colors.

For the LMC-OGLE3 objects, we make cross identification to the Spitzer point sources by using 'SAGE Winter 2008 IRAC Epoch 1 and Epoch 2 Catalog' and 'SAGE Winter 2008 MIPS $24 \mu \mathrm{m}$ Epoch 1 and Epoch 2 Catalog' by finding the nearest source within $5^{\prime \prime}$. For the SMC-OGLE3 objects, we make cross identification to Spitzer photometric data by using 'SAGE-SMC IRAC Epoch 0, Epoch 1, and Epoch 2 Catalog' and 'SAGE-SMC MIPS $24 \mu \mathrm{m}$ Epoch 0, Epoch 1, and Epoch 2 Catalog' by finding the nearest source within $5^{\prime \prime}$. These SAGE catalogs were extracted from the full list by placing strict restrictions on the source quality.

For the SAGE sample objects in the LMC and SMC, we use the Spitzer photometric data given in the references (Riebel et al. 2012; Srinivasan et al. 2016), which are from the SAGE Mosaic Photometry Archive. The original SAGE survey was conducted in two epochs spaced about three months apart (Meixner et al. 2006) and the observations from these epochs were combined into the single mosaic photometry archive, which is deeper and has smaller photometric errors.

Therefore, the Spitzer colors for the same object in the OGLE3 and SAGE samples can be slightly different because different catalogs were used (see IR 2CDs in Figures 7 - 9). Table 1 lists the sample AGB stars in the LMC and SMC and numbers of the cross-matched counterparts.

Figures 3 shows the comparison of the fluxes (in mag) at Spitzer and WISE bands for the cross-identified AGB stars in the LMC (OGLE3 and SAGE samples). The overall comparison is fairly consistent for most objects. Compared with OGLE3, there are less observed data at NIR bands but there are more observed data at MIR bands in the SAGE sample. This could be due to the different identification method for the SAGE sample (see Section 2.3), which would identify more optically invisible AGB stars with thick dust shells. The larger scatters at the S5[24] band for dimmer C-AGB stars would be due to the $\mathrm{Mg}_{0.9} \mathrm{Fe}_{0.1} \mathrm{~S}$ dust feature at $28 \mu \mathrm{m}$ (see Section 4.1).

\section{5. $O H / I R$ stars}

$\mathrm{OH} / \mathrm{IR}$ stars are generally considered to be more massive O-AGB stars with thicker dust envelopes and higher massloss rates. Chen et al. (2001) presented a list 1065 OH/IR stars in our Galaxy. The list has been corrected and updated (Suh \& Kwon 2011; Kwon \& Suh 2012; Suh \& Hong 2017), and a new list of $1520 \mathrm{OH} / \mathrm{IR}$ stars are included in the list of 3828 O-AGB stars in our Galaxy (Suh \& Hong 2017). On the IR 2CDs in Figures 4 and 5, the data for those Galactic $\mathrm{OH} / \mathrm{IR}$ stars are also plotted.

Only a small number of OH/IR stars are identified in the LMC and SMC yet. Goldman et al. (2017) presented a list of positively identified $10 \mathrm{OH} / \mathrm{IR}$ stars in the LMC. All of the 10 $\mathrm{OH} / \mathrm{IR}$ objects are included in the LMC-SAGE sample (see Table 1) and 6 objects from them are in the LMC-OGLE3 sample (Soszyński et al. 2009), which are classified as Miras.

There is no clear identification of OH/IR stars in the SMC yet (Goldman et al. 2018). Goldman et al. (2018) suspected that, compared with the OH/IR stars in the Galaxy and the LMC, the lower metallicity and star formation rate in the SMC may curtail the last dusty stellar wind phase of the most massive O-AGB stars (see Section 1).

\section{INFRARED TWO-COLOR DIAGRAMS}

We have complete or nearly complete SEDs from infrared spectroscopy only for a relatively small number of stars. A large number of stars have infrared photometric fluxes from the NIR to the FIR band thanks to the 2MASS, WISE, and Spitzer observations. Although the photometric fluxes are less useful than a full SED, the large number of observations at various wavelength bands can be used to form a 2CD, which can be compared with theoretical models. IR 2CDs are useful to statistically distinguish various properties of AGB 

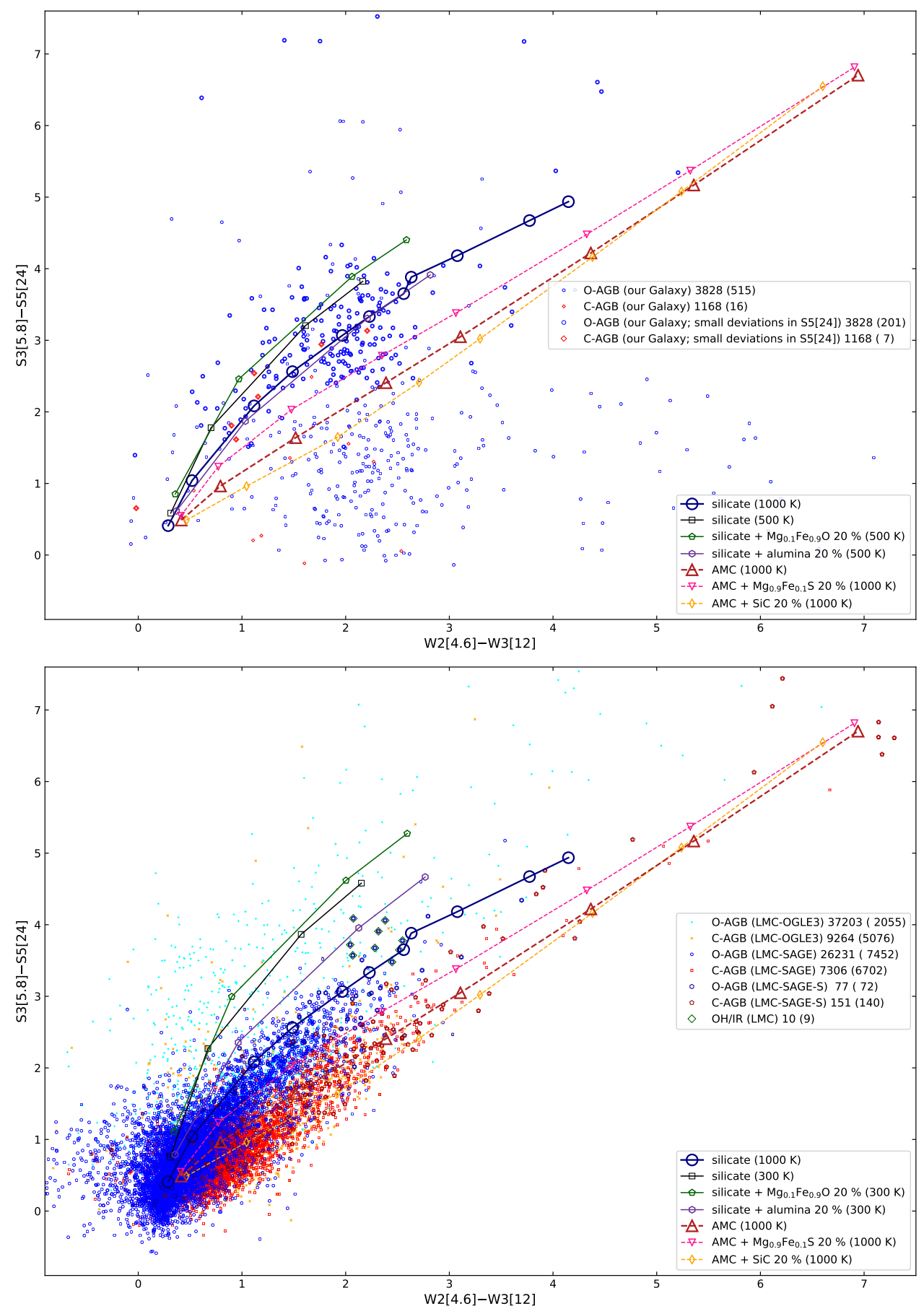

Figure 7. WISE-Spitzer 2CDs for AGB stars in our Galaxy and the LMC (OGLE3 and SAGE samples) compared with theoretical models (see Section 4). For O-AGB models (Silicate $T_{c}=1000 \mathrm{~K}$ ): $\tau_{10}=0.001,0.01,0.05,0.1,0.5,1,3,7,15,30$, and 40 from left to right. For C-AGB models (AMC $T_{c}=1000 \mathrm{~K}$ ): $\tau_{10}=0.001,0.01,0.1,0.5,1,2,3$, and 5 from left to right. For each class, the number of objects is shown. The number in parenthesis denotes the number of the plotted objects on the $2 \mathrm{CD}$ with good quality observed colors. 

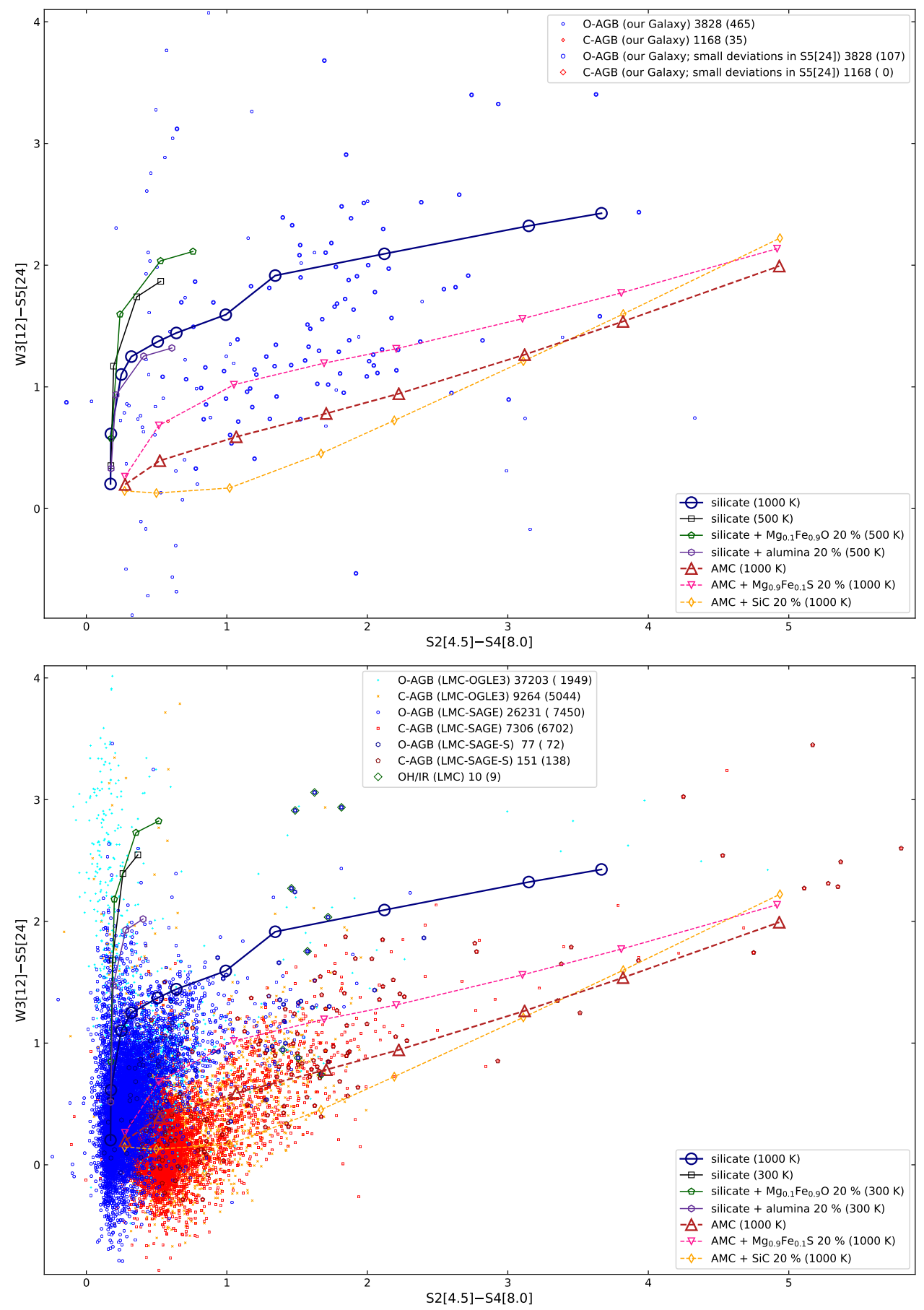

Figure 8. WISE-Spitzer 2CDs for AGB stars in our Galaxy and the LMC (OGLE3 and SAGE samples) compared with theoretical models (see Section 4). For O-AGB models (Silicate $T_{c}=1000 \mathrm{~K}$ ): $\tau_{10}=0.001,0.01,0.05,0.1,0.5,1,3,7,15,30$, and 40 from left to right. For C-AGB models (AMC $T_{c}=1000 \mathrm{~K}$ ): $\tau_{10}=0.001,0.01,0.1,0.5,1,2,3$, and 5 from left to right. For each class, the number of objects is shown. The number in parenthesis denotes the number of the plotted objects on the $2 \mathrm{CD}$ with good quality observed colors. 

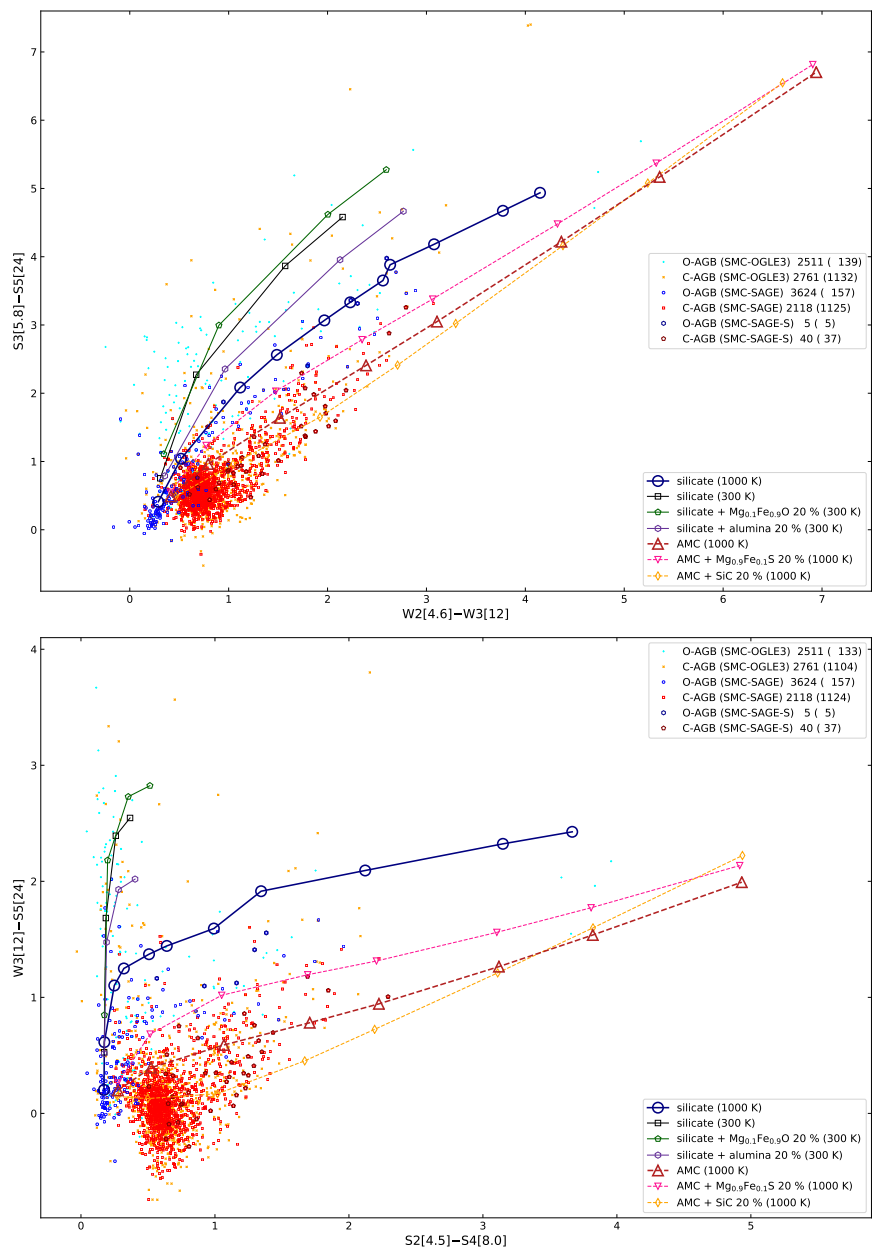

Figure 9. WISE-Spitzer 2CDs for AGB stars in the SMC (OGLE3 and SAGE sample) compared with theoretical models (see Section 4). For O-AGB models (Silicate $T_{c}=1000 \mathrm{~K}$ ): $\tau_{10}=0.001$, $0.01,0.05,0.1,0.5,1,3,7,15,30$, and 40 from left to right. For C-AGB models $\left(\right.$ AMC $\left.T_{c}=1000 \mathrm{~K}\right): \tau_{10}=0.001,0.01,0.1,0.5$, $1,2,3$, and 5 from left to right. For each class, the number of objects is shown. The number in parenthesis denotes the number of the plotted objects on the $2 \mathrm{CD}$ with good quality observed colors.

stars and we may use IR 2CDs to find new candidate objects for AGB stars (e.g., Suh \& Hong 2017).

Table 2 lists the IR bands used for the IR 2CDs presented in this work. In this work, we ignore reddening effects at all IR bands for all objects in our Galaxy and the Magellanic Clouds. Though the dereddening coefficient at the K[2.2] band for objects in the LMC is known to be small (about 0.0372 mag; Riebel et al. 2012), it could be larger for distant AGB stars in our Galaxy.

In this work, we use only good quality observational data at all wavelength bands for the 2MASS and WISE photometric data (quality A for 2MASS; quality A or B for WISE; see Section 2.1) for plotting IR 2CDs. For the Spitzer photometric data, we use all of the available data, which are from the good quality catalogs (see Sections 2.2 and 2.3).
For IR 2CDs, we use the three IR colors at shorter wavelength bands (W2[4.6]-W3[12], K[2.2]-W3[12], and $\mathrm{S} 2[4.5]-\mathrm{S} 4[8.0])$ for the horizontal axes. These colors are mostly affected by the dust grains in the inner dust shells so they are good measures of the overall dust optical depth. For the vertical axes, we use the three IR colors at longer wavelength bands (W3[12]-W4[22], S3[5.8]-S5[24], and W3[12]$\mathrm{S} 5[24])$ which are affected by the dust grains in more detached or outer dust shells.

Figures 4 - 9 show various IR 2CDs using the four different combinations of observed IR colors. We compare the observations with the theoretical dust shell models (see Section 4) for AGB stars in our Galaxy and the Magellanic Clouds. See Section 5 for comparison between theory and observations.

Figure 4 and 5 show the WISE 2CDs using W3[12]$\mathrm{W} 4[22]$ versus W2[4.6]-W3[12] and W3[12]-W4[22] versus $\mathrm{K}$ [2.2]-W3[12], respectively. The upper panels plot AGB stars in our Galaxy and the lower panels plot AGB stars in the LMC. Figure 6 shows the two 2CDs for AGB stars in the SMC.

Figures 7 and 8 show WISE-Spitzer 2CDs for AGB stars in our Galaxy and the LMC. Figure 7 shows 2CDs using S3[5.8]-S5[24] versus W2[4.6]-W3[12] and Figure 8 shows 2CDs using W3[12]-S5[24] versus S2[4.5]-S4[8.0]. Figure 9 shows the two 2CDs for AGB stars in the SMC. Note that the Spitzer colors for the same object in the OGLE3 and SAGE samples can be slightly different because different catalogs are used (see Section 2.4).

Generally, the stars that have thick dust shells with large dust optical depths are located in the upper-right regions on the IR 2CDs. But there can be some deviations. When the wavelength bands are near some dust or gas features (e.g., the silicate dust feature at $10 \mu \mathrm{m}$; see Section 4.2), the IR colors can be severely affected.

Though both OGLE3 and SAGE sample stars show similar properties on the IR 2CDs, the SAGE sample can be regarded as more reliable sample of AGB stars because the selection method was more sophisticated and considered more NIR and MIR photometric data (see Section 2.3).

Figure 10 shows the error bar plots of the averaged observed colors of the sample stars for various IR colors used for the four IR 2CDs presented in Figures 4 - 9. For the objects in the Magellanic Clouds, we present only the SAGE sample stars.

On all of the IR 2CDs, we also plot the sequences of theoretical dust shell models at increasing dust optical depth for AGB stars (see Section 4). We will discuss the meanings of these 2CDs in Section 5 by comparing the observations with the theoretical models.

\section{THEORETICAL DUST SHELL MODELS}

On all of the 2CDs in Figures 4 - 9, theoretical model tracks for AGB stars are plotted to be compared with the observations. We use spherically symmetric dust shell models for AGB stars.

\subsection{Dust Shell Models for AGB Stars}



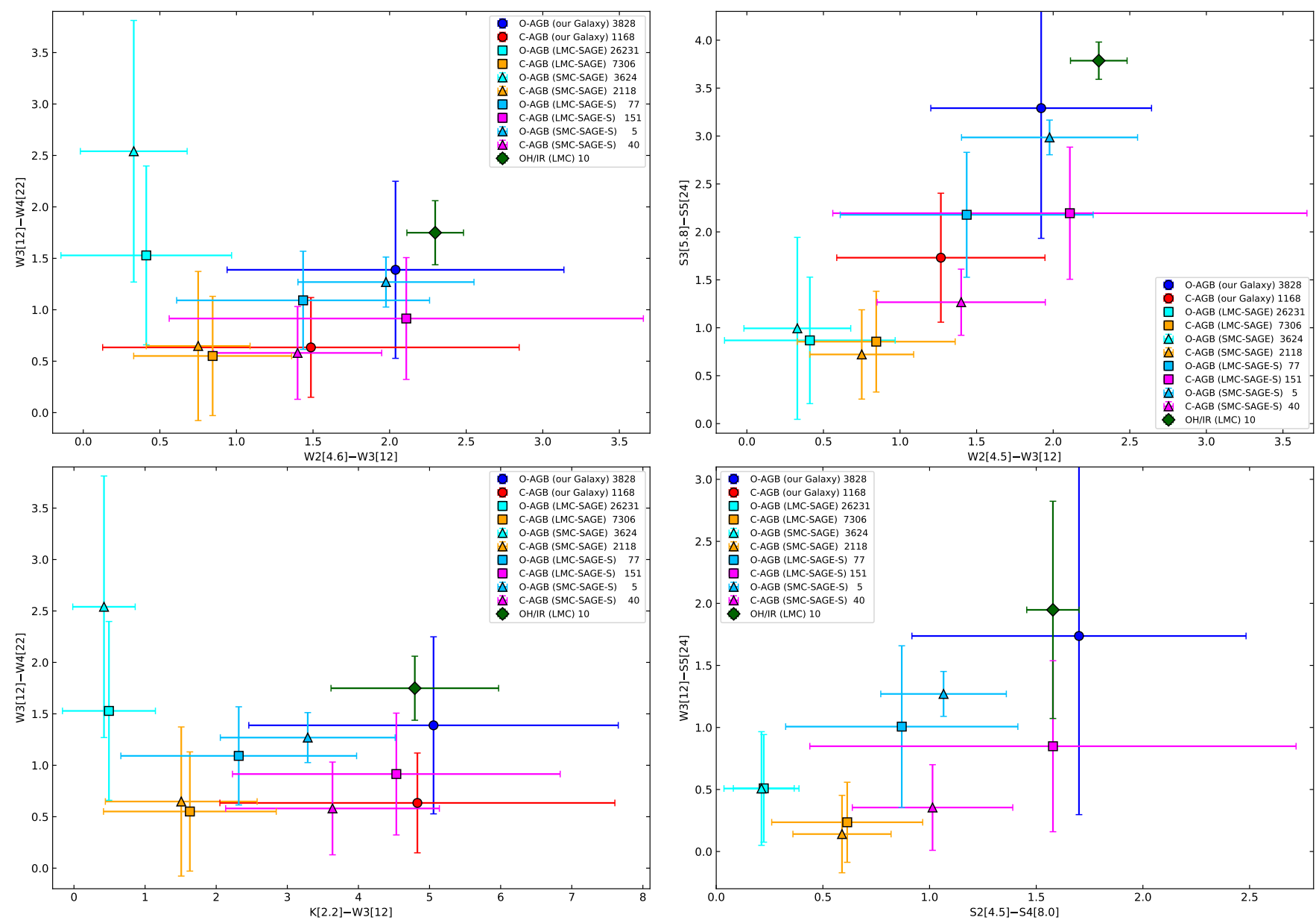

Figure 10. Averaged observed colors for sample stars (see Table 1) used for the IR 2CDs (see Figures 3 - 8). We plot only SAGE sample stars for the Magellanic Clouds. For the AGB stars in our Galaxy using Spitzer data, we consider only the objects with small deviations in the S5[24] flux (see Section 2.2).

We use the radiative transfer code DUSTY (Ivezić \& Elitzur 1997) for a spherically symmetric dust shell. For all models, we use a continuous power law $\left(\rho \propto r^{-2}\right)$ dust density distribution. We assume that the dust formation temperature $\left(T_{c}\right)$ is $1000 \mathrm{~K}$. But for LMOA stars, we also use $500 \mathrm{~K}$ and $300 \mathrm{~K}$ because it is known that LMOA stars have lower $T_{c}$ (e.g., Suh 2004). The inner radius of the dust shell is set by the $T_{c}$ and the outer radius of the dust shell is taken to be $10^{4}$ times the inner radius. The radii of spherical dust grains are assumed to be $0.1 \mu \mathrm{m}$ uniformly. We use $10 \mu \mathrm{m}$ as the fiducial wavelength of the dust optical depth $\left(\tau_{10}\right)$. Because the shape of the model SED is independent on the luminosity of the central star when all other parameters are fixed, the DUSTY code calculates the model SED only in relative scale.

For O-AGB stars, we use optical constants of warm and cold silicate dust from Suh (1999). We compute eleven models $\left(\tau_{10}=0.001,0.01,0.05,0.1,0.5,1,3,7,15,30\right.$, and 40$)$ with $T_{c}=1000 \mathrm{~K}$. We use warm silicate for LMOA stars (7 models with $\tau_{10} \leq 3$ ) and cold silicate for HMOA stars (4 models $\tau_{10}>3$ ). We assume that the stellar blackbody tem- perature is $3000 \mathrm{~K}$ for $\tau_{10} \leq 0.1$ (four models), $2500 \mathrm{~K}$ for $0.1<\tau_{10} \leq 3$ (three models), and $2000 \mathrm{~K}$ for $\tau_{10}>3$ (four models). Figure 10 shows model SEDs for O-AGB stars (silicate; $T_{c}=1000 \mathrm{~K}$ ) for six major dust optical depths.

Only for LMOA stars with thin dust shells (the four models with $\left.\tau_{10} \leq 0.1\right)$, we also use lower $T_{c}(500 \mathrm{~K}$ and $300 \mathrm{~K})$. Also alumina and $\mathrm{Fe}-\mathrm{Mg}$ oxide grains as well as silicates are necessary to reproduce the observed SEDs (see Section 1). We use three different dust opacity models: a simple mixture of warm silicate and $\mathrm{Fe}_{0.9} \mathrm{Mg}_{0.1} \mathrm{O}$ (20\% by number) and a simple mixture of warm silicate and alumina (20\% by number) as well as pure warm silicate. For $\mathrm{Fe}_{0.9} \mathrm{Mg}_{0.1} \mathrm{O}$ dust, we use the optical constants from Henning et al. (1995). For alumina dust, we use the optical constants from Suh (2016), which were derived from the optical constants in narrower wavelength range obtained by Begemann et al. (1997).

For C-AGB stars, we use the optical constants of AMC and SiC dust grains from Suh (2000) and Pégourié (1988), respectively. For $\mathrm{MgS}$ dust, we use the optical constants of $\mathrm{Mg}_{0.9} \mathrm{Fe}_{0.1} \mathrm{~S}$ dust, which is close to pure $\mathrm{MgS}$, from Begemann et al. (1994). We compute eight models $\left(\tau_{10}=0.001\right.$, 

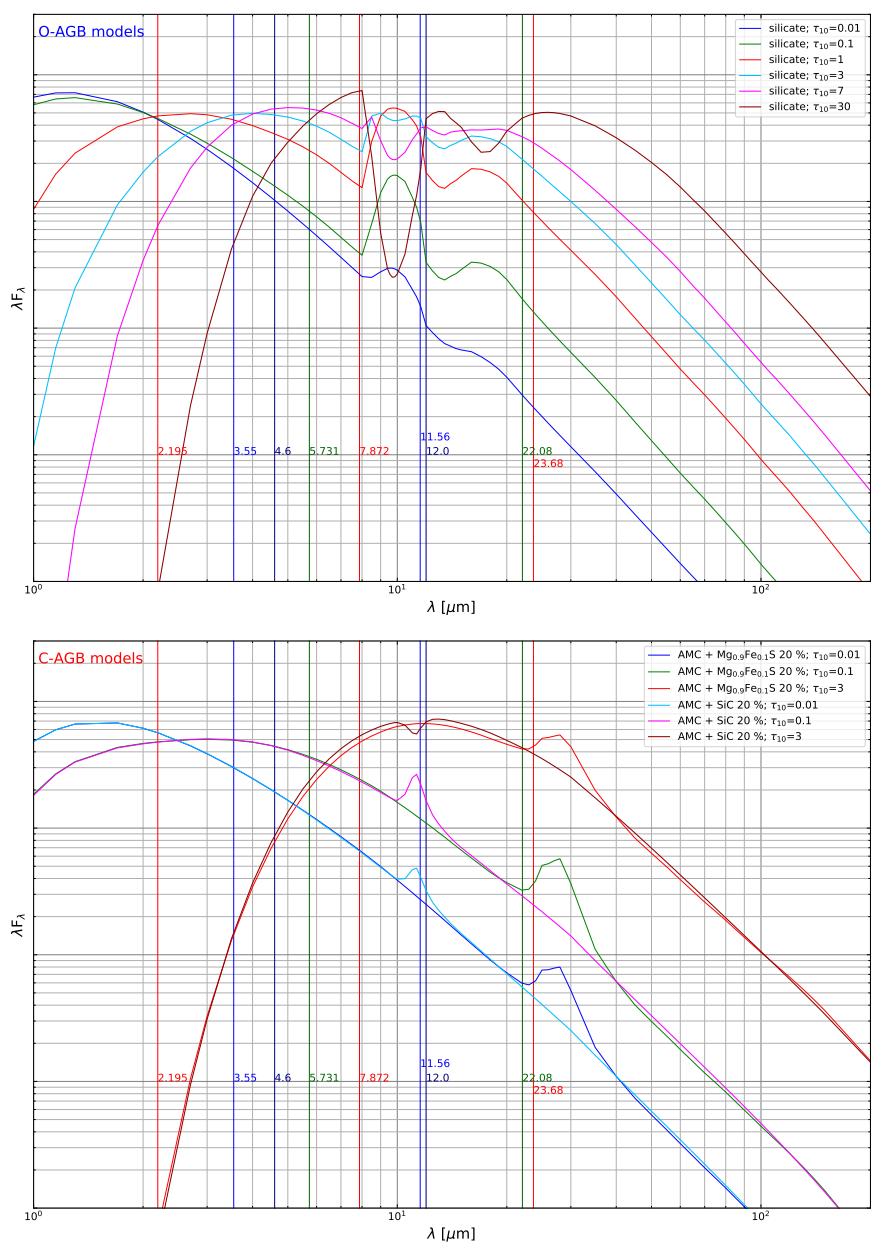

Figure 11. Theoretical model SEDs for O-AGB stars (silicate; $\left.T_{c}=1000 \mathrm{~K}\right)$ and C-AGB stars $\left(\mathrm{AMC} ; T_{c}=1000 \mathrm{~K}\right)$ for a number of dust optical depths (see 4.1). The reference wavelengths for major IR bands are also indicated (see Table 2).

$0.01,0.1,0.5,1,2,3$, and 5) with $T_{c}=1000 \mathrm{~K}$. We assume that the stellar blackbody temperature is $2500 \mathrm{~K}$ for $\tau_{10}<1$ (four models) and $2000 \mathrm{~K}$ for $\tau_{10} \geq 1$ (four models). We use three different dust opacity models: a simple mixture of AMC and $\mathrm{Mg}_{0.9} \mathrm{Fe}_{0.1} \mathrm{~S}$ (20\% by number) and a simple mixture of AMC and $\mathrm{SiC}$ ( $10 \%$ by number) as well as pure AMC.

Figure 11 shows model SEDs for AGB stars $\left(T_{c}=1000 \mathrm{~K}\right)$ for major dust optical depths. For O-AGB models, silicate dust features at 10 and $18 \mu \mathrm{m}$ are shown for various dust optical depths $\left(\tau_{10}\right)$. For LMOA stars, amorphous alumina and $\mathrm{Fe}_{0.9} \mathrm{Mg}_{0.1} \mathrm{O}$ dust grains produce broad emission features at 11.8 and $19.6 \mu \mathrm{m}$, respectively (see Suh 2018). For C-AGB models, $\mathrm{SiC}$ dust features at $11.3 \mu \mathrm{m}$ and $\mathrm{Mg}_{0.9} \mathrm{Fe}_{0.1} \mathrm{~S}$ dust features at $28 \mu \mathrm{m}$ are shown for different dust optical depths.

The gas-to-dust ratio $(\Psi)$ is generally estimated to be 50 200 in our Galaxy (the average $\Psi$ is about 100) and $\Psi$ tends to decrease for a higher metallicity (Draine et al. 2007). Nanni et al. (2019) found that $\Psi$ is larger in the Magellanic Clouds ( $\Psi \sim 700$ ) probably due to the lower metallicity. The optical
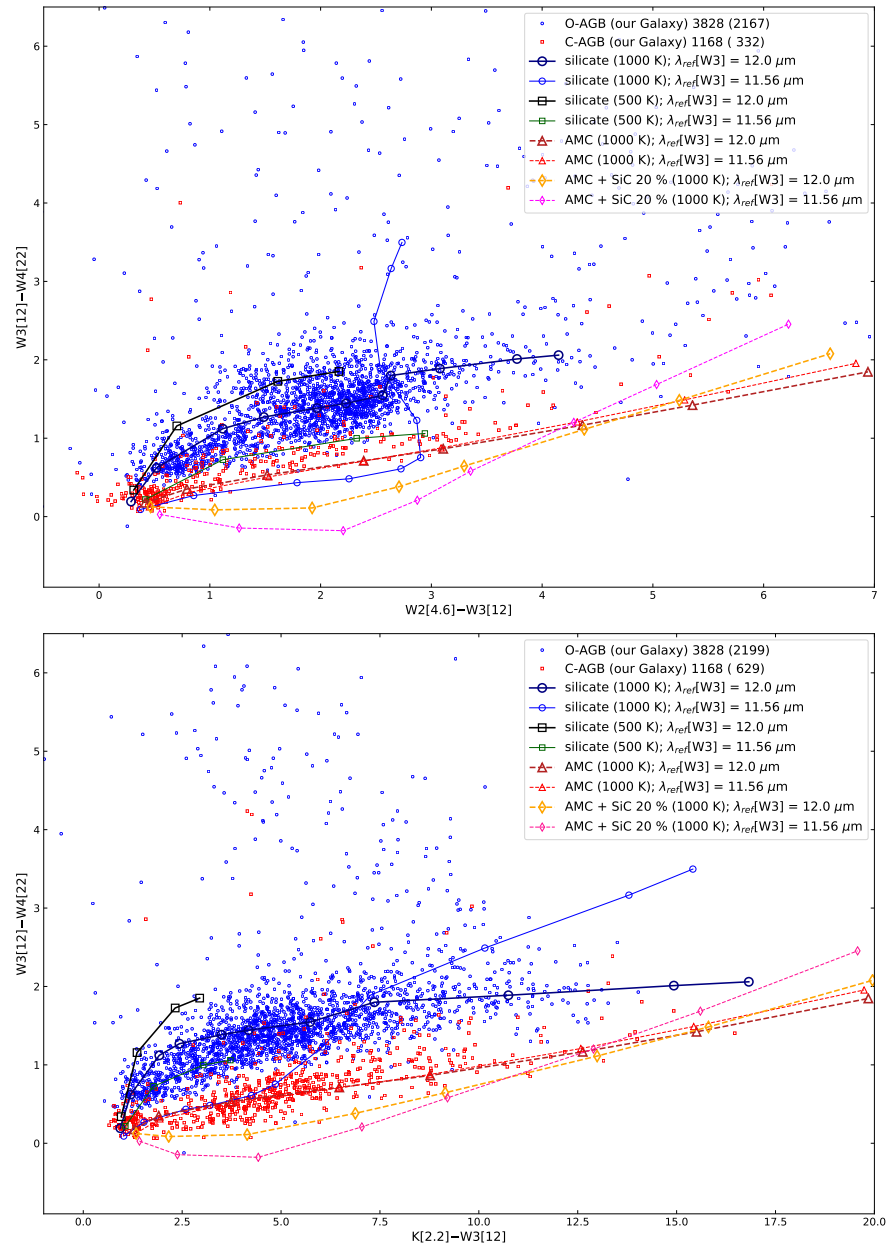

Figure 12. Theoretical dust shell model tracks on the WISE2MASS 2CDs for different reference wavelengths of the W3[12] band (see the model SEDs: Figure 11). These are compared with the observations of AGB stars in our Galaxy. In this work, we use the reference wavelength of $12 \mu \mathrm{m}$ for the WISE W3[12] band (see Table 2). This choice produces model colors that make a better fit with the observations of AGB stars in our Galaxy and the Magellanic Clouds (compare with Figures 4 - 9). See Section 4.2.

depths of the dust shells around AGB stars would be dependent on the initial masses and metallicity (e.g., Ventura et al. 2016). In a galaxy with a higher metallicity (and a lower $\Psi$ ), the star formation in the higher mass range would be more active and the galaxy would have a higher ratio of AGB stars with thick dust shell.

If we assume that the stellar blackbody luminosity is $10^{4} L_{\odot}, \Psi=100$, and dust shell expansion velocity is 15 $\mathrm{km} / \mathrm{sec}$, the mass-loss rates are $3.8 \times 10^{-7}, 7.1 \times 10^{-6}$, and $6.5 \times 10^{-5} M_{\odot} / y r$ for LMOA stars $\left(T_{c}=1000 \mathrm{~K} ; \tau_{10}=0.1\right)$, C-AGB stars with $\tau_{10}=1$, and HMOA star with $\tau_{10}=15$, respectively.

\subsection{Model color indices}


To compare theoretical models with observations on 2CDs, we need to obtain model colors from the model SEDs. We obtain the model colors using the reference (or effective or isophotal) wavelength and zero magnitude flux (ZMF) given in the reference for the 2MASS, WISE, and Spitzer photometric data (see Table 2).

The color index is defined by

$$
M_{\lambda 1}-M_{\lambda 2}=-2.5 \log _{10} \frac{F_{\lambda 1} / Z M F_{\lambda 1}}{F_{\lambda 2} / Z M F_{\lambda 2}}
$$

where $Z M F_{\lambda i}$ is the ZMF at given wavelength $(\lambda i)$ (see Table 2).

The reference wavelength for the W3[12] band largely affects the model colors for O-AGB stars because the wavelength is very near the conspicuous $10 \mu \mathrm{m}$ silicate features of the model SEDs (see Figure 10). For the WISE W3[12] band, the isophotal wavelength is $11.56 \mu \mathrm{m}$ (Jarrett et al. 2011) and the response function weighted average wavelength is 12.33 $\mu \mathrm{m}$. The isophotal wavelength $(11.56 \mu \mathrm{m})$ for the W3[12] band, which was obtained from the observations of Vega (Jarrett et al. 2011), could be too short for dusty AGB stars.

The theoretical model for O-AGB stars used in this work reproduced various spectral and photometric observations of O-AGB stars (e.g., Suh 2002; Suh 2004; González-Lópezlira 2018) in wide wavelength ranges reasonably well. However, the same model produced model colors that show very large deviations from the observed colors of O-AGB stars in our Galaxy (Suh 2018) and the Magellanic Clouds (this work) when we use $\lambda_{\text {ref }}=11.56 \mu \mathrm{m}$ for the W3[12] band.

For the WISE W3[12] band, we use the reference wavelength of $12 \mu \mathrm{m}$ and ZMF of $28.3 \mathrm{Jy}$ (the same values as those for the IRAS [12] band; Beichman et al. 1988) to obtain the theoretical model colors (see Table 2).

Figure 12 shows IR 2CDs using two different reference wavelengths for the W3[12] band compared with the observations of AGB stars in our Galaxy. This small change makes large differences for the O-AGB model colors. Though the $\mathrm{SiC}$ dust feature at $11.3 \mu \mathrm{m}$ is also affected, the effect is minor for C-AGB models without SiC dust (see Figure 11). With this choice $\left(\lambda_{r e f}=12 \mu \mathrm{m}\right.$ for the W3[12] band), the model colors fit the observed colors much better on all IR 2CDs for AGB stars in our Galaxy and the Magellanic Clouds (see Figures 3 - 8).

\subsection{Limitations of the Theoretical Models}

The theoretical dust shell model used in this work does not consider gas-phase radiation processes (see Section 4). AGB stars show various gas-phase emission or absorption features in NIR and MIR band due to circumstellar molecules such as $\mathrm{H}_{2} \mathrm{O}, \mathrm{CO}$, and $\mathrm{C}_{2} \mathrm{H}_{2}$ (e.g, Lançon \& Wood 2000; Le Bertre 2005; Gonneau et al. 2016). The deviations of the theoretical models from the observations would be larger at the wavelength bands where gas-phase radiation processes are more active.

Also, the spherically symmetric dust shell model does not consider nonspherical dust envelopes. The observed colors of
AGB stars with nonspherical dust envelopes can show various deviations from the theoretical models at NIR and MIR bands.

\section{COMPARISON BETWEEN THEORY AND OBSERVATIONS}

On various IR 2CDs using four different combinations of IR colors in Figures 4 - 9, we compare the observations with the theoretical dust shell models (see Section 4) for AGB stars. We find that the theoretical dust shell model can roughly reproduce the observations of AGB stars on the IR $2 \mathrm{CDs}$ using the dust opacity functions of amorphous silicate and amorphous carbon with a mixture of other dust species.

Compared with our Galaxy, we find that more AGB stars in the LMC and SMC are located in the lower-left regions of any IR 2CDs. For all of the observed colors (W2[4.6]W3[12], W3[12]-W4[22], K[2.2]-W3[12], S2[4.5]-S4[8.0], S3[5.8]-S5[24], and W3[12]-S5[24]), the averaged color of AGB stars in the LMC or SMC is bluer than that of AGB stars in our Galaxy (see Figure 10). Note that this difference is systematic only for the averaged K[2.2]-W3[12] color of the O-AGB stars, and the error bars overlap for all other colors.

Though the methods of chemical classification into $\mathrm{O}$ or $\mathrm{C}$ for the two AGB samples of the Magellanic Clouds (OGLE3 and SAGE) are different, only a minor portion of objects are classified into different classes (see Section 2.3). The identification and chemical classification for the SAGE samples would be more reliable because they are based on the comparison of the more photometric data at NIR and MIR bands with the Grid of AGB and RSG ModelS (GRAMS) from Riebel et al. (2012) and Srinivasan et al. (2016). The SAGES samples of AGB stars would be even more reliable because they were obtained using the Spitzer IRS spectral data. For the comparison of number distributions of AGB stars on the IR 2CDs with the theoretical models, we use only the SAGE samples (including the SAGE-S samples) for the objects in the Magellanic Clouds.

\subsection{Number distributions of IR colors}

We may compare the number distribution of observed IR colors with the theoretical model. Figure 13 shows the number density distributions of the four observed IR colors for AGB stars in our Galaxy and the Magellanic Clouds. The four IR colors (W2[4.6]-W3[12], K[2.2]-W3[12], S2[4.5]$\mathrm{S} 4[8.0]$, and S3[5.8]-S5[24]) are good measures of the dust optical depth $\left(\tau_{10}\right)$ as we can see on the IR 2CDs (see Figures 4 - 9).

We mark the theoretical model colors on Figure 13. For OAGB stars, the dust shell (silicate; $T_{c}=1000 \mathrm{~K}$ ) model colors for typical LMOA stars $\left(\tau_{10}=0.1\right)$ and HMOA stars $\left(\tau_{10}=7\right.$ and 15) are indicated. For C-AGB stars, the dust shell (AMC; $\left.T_{c}=1000 \mathrm{~K}\right)$ model colors for the thin dust shell $\left(\tau_{10}=0.1\right)$ and thick dust shell $\left(\tau_{10}=1\right)$ model colors are indicated. See Section 4.1 for the detailed model parameters.

We may also obtain the percentages of the objects with thin or thick dust shells using the information presented in Figure 13. Table 3 lists the percentages of the objects with 

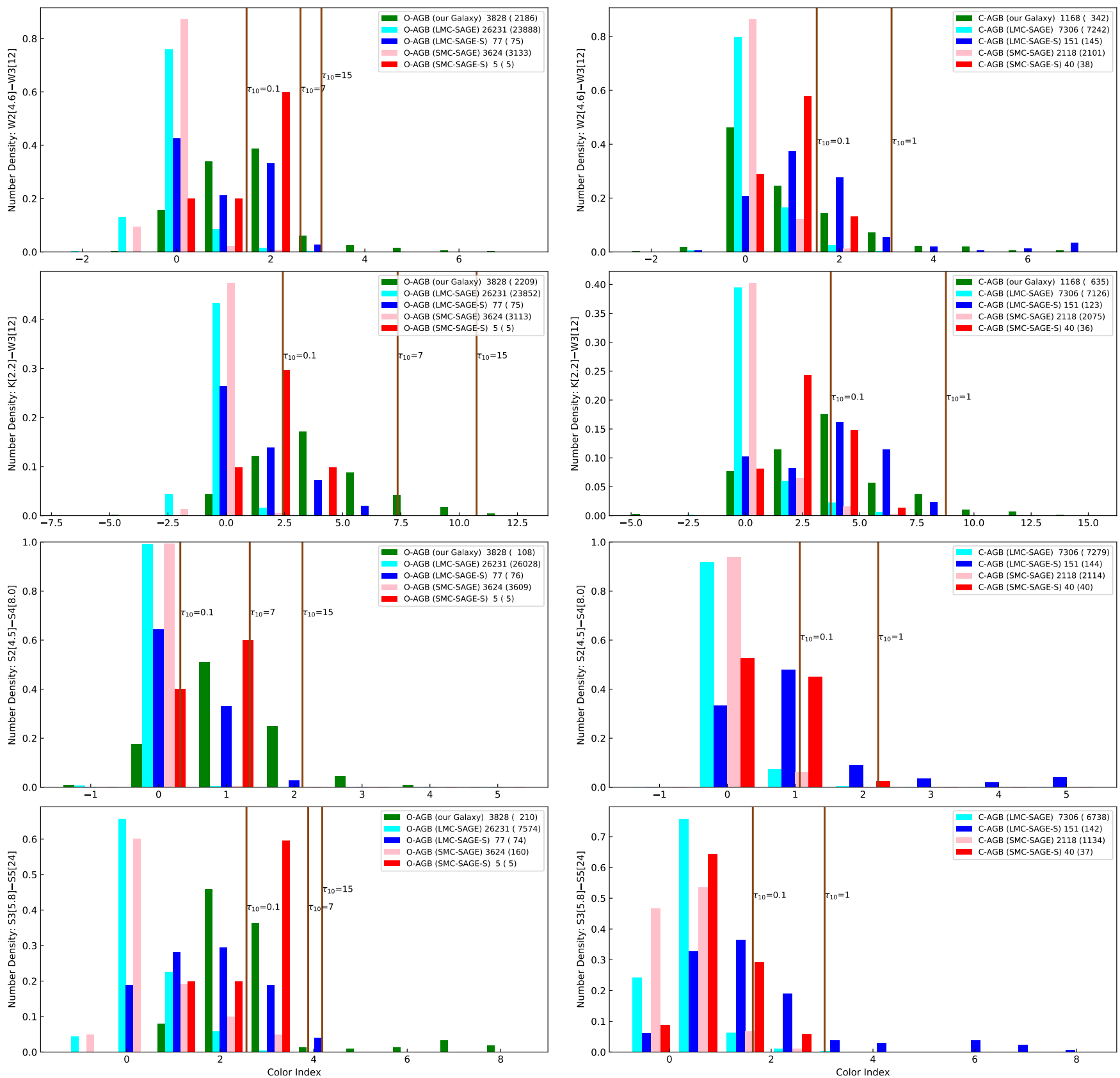

Figure 13. Number density distributions of observed IR colors for O-AGB and C-AGB stars in our Galaxy and the Magellanic Clouds (LMCSAGE and SMC-SAGE). The vertical brown lines indicate theoretical model colors for the two or three dust shell optical depths $\left(\tau_{10}\right)$. For each class, the number of objects is also shown. The number in parenthesis denotes the number of the plotted objects with good quality observed colors. See Table 3.

thin (O-AGB: $\tau_{10}<0.1$; C-AGB: $\tau_{10}<0.1$ ), thick (O-AGB: $\tau_{10}>7$; C-AGB: $\tau_{10}>1$ ), and very thick (O-AGB: $\tau_{10}>$ 15) dust shells from the comparison of the observed colors with the theoretical models for the seven IR colors: W2[4.6]W3[12], K[2.2]-W3[12], S1[3.6]-S4[8.0], S2[4.5]-S4[8.0], S3[5.8]-S5[24], IRAS [12]-[25], and 2MASS-IRAS K-[12]. Though the three colors (S1[3.6]-S4[8.0], IRAS [12]-[25], and 2MASS-IRAS K-[12]) were not used for the IR 2CDs presented in this paper, they are also good measures of the dust optical depth. The data for IRAS [12]-[25] and 2MASSIRAS K-[12] colors are from Suh \& Hong (2017), which presented IR properties of AGB stars in our Galaxy.

Table 4 lists weighted averaged percentages of the objects with thick dust shells (O-AGB: $\tau_{10}>7$; C-AGB: $\tau_{10}>1$; O-AGB: $\tau_{10}>15$ ) obtained from Table 3. For AGB stars in our Galaxy, we obtain the weighted averaged percentages from the data for four IR colors: W2[4.6]-W3[12], K[2.2]W3[12], IRAS [12]-[25], and 2MASS-IRAS K-[12]. We do 
Table 3. Percentages of the objects with thin and thick dust shells from the comparison of the observed colors with the theoretical models. See Figure 13.

\begin{tabular}{llllllll}
\hline \hline Class $^{1}$ & W2[4.6]-W3[12] & K[2.2]-W3[12] & S1[3.6]-S4[8.0] & S2[4.5]-S4[8.0] & S3[5.8]-S5[24] & [12]-[25] $^{2}$ & K-[12] $^{2}$ \\
\hline O-AGB (our Galaxy) & $29.0,17.5(2186)$ & $14.4,17.6(2209)$ & $3.7,39.7(136)$ & $2.8,67.6(108)$ & $21.9,13.8(210)$ & $45.2,13.8(3568)$ & $8.4,21.9(2189)$ \\
O-AGB (LMC-SAGE) & $95.1,0.7(23888)$ & $98.4,0.01(23852)$ & $97.9,0.04(25907)$ & $85.2,0.2(26028)$ & $97.6,0.1(7574)$ & - & - \\
O-AGB (LMC-SAGE-S) & $52.0,5.3(75)$ & $64.0,0.0(75)$ & $40.5,5.4(74)$ & $14.5,29.0(76)$ & $59.5,8.1(74)$ & - & - \\
O-AGB (SMC-SAGE) & $98.3,0.2(3133)$ & $99.2,0.0(3113)$ & $98.7,0.1(3610)$ & $90.0,0.3(3609)$ & $91.9,0.6(160)$ & - & - \\
O-AGB (SMC-SAGE-S) & $20.0,0.0(5)$ & $20.0,0.0(5)$ & $0.0,0.0(5)$ & $0.0,20.0(5)$ & $40.0,20.0(5)$ & - & - \\
\hline C-AGB (our Galaxy) & $61.7,12.0(342)$ & $33.7,8.5(635)$ & - & - & - & $52.7,23.0(1098)$ & $22.3,18.2(687)$ \\
C-AGB (LMC-SAGE) & $92.2,0.6(7242)$ & $92.3,0.06(7126)$ & $93.3,0.6(7247)$ & $92.8,0.6(7279)$ & $94.0,0.6(6738)$ & - & - \\
C-AGB (LMC-SAGE-S) & $37.2,13.1(145)$ & $34.2,1.6(123)$ & $37.1,13.3(143)$ & $36.8,13.9(144)$ & $40.9,14.8(142)$ & - & - \\
C-AGB (SMC-SAGE) & $95.3,0.0(2101)$ & $93.8,0.0(2075)$ & $95.7,0.1(2115)$ & $95.3,0.1(2114)$ & $94.4,0.2(1134)$ & - & - \\
C-AGB (SMC-SAGE-S) & $60.5,0.0(38)$ & $47.2,0.0(36)$ & $55.0,2.5(40)$ & $55.0,2.5(40)$ & $78.4,2.7(37)$ & - & - \\
\hline O-AGB (our Galaxy) & $10.1(2186)$ & $2.8(2209)$ & $11.0(136)$ & $22.2(108)$ & $8.1(210)$ & $9.2(3568)$ & $4.8(2189)$ \\
O-AGB (LMC-SAGE) & $0.37(23888)$ & $0.004(23852)$ & $0.0(25907)$ & $0.008(26028)$ & $0.04(7574)$ & - & - \\
O-AGB (LMC-SAGE-S) & $2.67(75)$ & $0.0(75)$ & $0.0(74)$ & $1.32(76)$ & $1.35(74)$ & - & - \\
O-AGB (SMC-SAGE) & $0.064(3133)$ & $0.0(3113)$ & $0.055(3610)$ & $0.055(3609)$ & $0.0(160)$ & - & - \\
O-AGB (SMC-SAGE-S) & $0.0(5)$ & $0.0(5)$ & $0.0(5)$ & $0.0(5)$ & $0.0(5)$ & - & - \\
\hline
\end{tabular}

For each column of the IR color, the percentages of the objects with thin and thick dust shells for O-AGB stars $\left(\tau_{10}<0.1, \tau_{10}>7\right)$ and C-AGB stars $\left(\tau_{10}<0.1, \tau_{10}>1\right)$ are listed except for the last five rows. The last five rows (marked by ${ }^{*}$ ) list the percentages of the O-AGB stars with very thick dust shells $\left(\tau_{10}>15\right)$. The number in parenthesis denotes the number of the observed objects used for the IR 2CDs and histograms. For the colors of AGB stars in our Galaxy using Spitzer data, we consider only the objects with small deviations in the S5[24] flux (see Section 2.2). ${ }^{1}$ See Table 1 for the sample information. ${ }^{2}$ The data for the IRAS-2MASS colors are from Suh \& Hong (2017).

Table 4. Weighted averaged percentages ${ }^{1}$ of AGB stars with thin and thick dust shells

\begin{tabular}{lllll}
\hline \hline Class & Dust Shell & Our Galaxy & LMC & SMC \\
\hline O-AGB & silicate $\left(\tau_{10}\right)<0.1$ & $27.1 \%$ & $79.5 \%$ & $82.7 \%$ \\
O-AGB & silicate $\left(\tau_{10}\right)>7$ & $17.2 \%$ & $3.4 \%$ & $1.8 \%$ \\
C-AGB & AMC $\left(\tau_{10}>1\right)$ & $17.1 \%$ & $9.4 \%$ & $1.3 \%$ \\
O-AGB & silicate $\left(\tau_{10}>15\right)$ & $7.0 \%$ & $0.44 \%$ & $0.048 \%$ \\
\hline
\end{tabular}

${ }^{1}$ The averages are weighted by the numbers of observed objects listed in Table 3 (see the text for details). For the Magellanic clouds, the numbers of the observed objects in SAGE-S samples are multiplied by 100 .

not use Spitzer colors for our Galaxy because the sample number is small and they show large deviations.

For AGB stars in the Magellanic Clouds, we obtain the weighted averaged percentages from the data for four IR colors: W2[4.6]-W3[12], S1[3.6]-S4[8.0], S2[4.5]-S4[8.0], S3[5.8]-S5[24]. We do not consider K[2.2]-W3[12] color because it shows 'bluing effect' for the Magellanic Clouds (see Section 5.3). In obtaining the weighted averaged percentages for the objects in the Magellanic Clouds, the numbers of observed objects for SAGE-S samples are multiplied by 100 because the SAGE-S samples are more reliable than the SAGE samples.

Compared with our Galaxy, we find that the LMC and SMC are deficient in O-AGB stars with thick dust shells on any IR 2 CDs. The weighted averaged percentages of HMOA stars with thick dust shells $\left(\tau_{10}>7\right)$ for our Galaxy $(17.2$ $\%)$ is larger than the ones for the LMC (3.4\%) and SMC $(1.8 \%)$ SAGE sample stars (see Table 4$)$. The percentages of HMOA stars with very thick dust shells $\left(\tau_{10}>15\right)$ for our Galaxy (7.0\%) is even larger than the ones for the LMC $(0.44 \%)$ and $\operatorname{SMC}(0.348 \%)$.

For C-AGB stars in our Galaxy and the LMC, the observations can be reproduced by the $\mathrm{C}$-AGB models in wide ranges of the dust optical depth $\left(\tau_{10}=0.001-5\right)$ on all IR 2CDs except for the 2CD using the K[2.2]-W3[12] color, for which AGB stars in the LMC show the 'bluing' effect (see Section 5.3). The weighted averaged percentages of C-AGB stars with thick dust shells $\left(\tau_{10}>1\right)$ for our Galaxy $(17.1 \%)$ is larger than the ones for the LMC $(9.4 \%)$ and SMC $(1.3 \%)$ (see Table 4).

Compared with our Galaxy, we find that much larger portions of O-AGB stars in the LMC and SMC have thin dust shells with smaller dust optical depths $\left(\tau_{10}\right)$ on the IR 2CDs (see Figures 4 - 9 and histograms in Figure 13). The weighted averaged percentages of LMOA stars with thin dust shells $\left(\tau_{10}<0.1\right)$ for the LMC $(79.5 \%)$ or SMC $(82.7 \%)$ are much larger than the ones for our Galaxy (27.1\%) (see Table 4). This could be due to a selection effect. In our Galaxy, it is difficult to identify the optically visible AGB stars (with thin dust shells) using optical or NIR surveys because of the severe extinction by the Galactic disk. Note that the sample of Galactic AGB stars is based on the IRAS PSC (see Section 2.2).

\subsection{WISE 2CDs}

Figure 4 shows WISE 2CDs using W3[12]-W4[22] versus W2[4.6]-W3[12]. The upper panel plots AGB stars in our Galaxy and the lower panel plots AGB stars in the LMC.

For LMOA stars with thin dust shells in our Galaxy and the LMC, the silicate dust with a mixture of amorphous alumina $\left(\mathrm{Al}_{2} \mathrm{O}_{3}\right)$ and $\mathrm{Fe}_{0.9} \mathrm{Mg}_{0.1} \mathrm{O}$ can explain wider regions on the IR 2CDs. Compared with our Galaxy, we find that LMOA stars in the LMC have more detached dust shells ( $\left.T_{c} 300 \mathrm{~K}\right)$. 
For O-AGB stars, the ratio of HMOA stars with thick dust shells $\left(\tau_{10}>7\right)$ for the LMC (SAGE-S: $5.3 \%$; SAGE: 0.68 $\%$ ) is smaller than the one for our Galaxy $(17.5 \%)$ (see Table 3). Even for the most dusty O-AGB stars (or OH/IR stars) in the LMC, $\tau_{10}$ would be about $1-7$, which produce conspicuous emission or shallow self absorption silicate features at $10 \mu \mathrm{m}$ (see Figure 10; see Jones et al. 2017 for the Spitzer IRS spectra). On the other hand, $\tau_{10}$ for many O-AGB stars in our Galaxy are about 10-40, which produce deep silicate absorption features at $10 \mu \mathrm{m}$ (e.g., Suh 1999; Suh 2004).

C-AGB stars in our Galaxy and the LMC are located in the wide range of the $\mathrm{C}$-AGB model colors $\left(\tau_{10}=0.001-5\right)$ on this 2CD. For C-AGB stars, the ratio of C-AGB stars with thick dust shells $\left(\tau_{10}>1\right)$ for the LMC (SAGE-S: $13.1 \%$; SAGE: $0.6 \%)$ is comparable to the one for our Galaxy (12.0 $\%$ ) (see Table 3). For C-AGB stars, the effect of SiC dust feature (at $11.3 \mu \mathrm{m}$ ) on the W3[12]-W4[22] color is conspicuous while the effect of $\mathrm{Mg}_{0.9} \mathrm{Fe}_{0.1} \mathrm{~S}$ dust feature (at $26 \mu \mathrm{m}$ ) is small.

The upper panel of Figure 6 shows the WISE 2CDs for AGB stars in the SMC. For C-AGB stars, the ratio of objects with thick dust shells $\left(\tau_{10}>1\right)$ for the SMC is $0 \%$, which is much smaller than those for the LMC and our Galaxy (see Table 3).

\subsection{WISE-2MASS $2 C D s$}

Figure 5 shows WISE-2MASS 2CDs using W3[12]W4[22] versus K[2.2]-W3[12]. The upper panel plots AGB stars in our Galaxy and the lower panel plots AGB stars in the LMC.

Unlike other three IR 2CDs (compare with Figures 4 9), we find that the observed K[2.2]-W3[12] colors on this WISE-2MASS 2CD are bluer than the theoretical model colors. Compared with other IR colors, the observed K[2.2]W3[12] colors show smaller percentages of thick dust shell for both the O-AGB and C-AGB stars in our Galaxy and the LMC (see Table 3). The effect is much stronger for the AGB stars in the LMC than for those in our Galaxy. For the LMCSAGE-S sample stars, the ratio of C-AGB stars with thick dust shells $\left(\tau_{10}>1\right)$ for the K[2.2]-W3[12] color $(1.6 \%)$ is much smaller than those for other colors (13.1-14.8\%) and the ratio of O-AGB stars with thick dust shells $\left(\tau_{10}>7\right)$ for the $\mathrm{K}[2.2]-\mathrm{W} 3[12]$ color $(0.0 \%)$ is also much smaller than those for other colors (5.3-29.0\%).

The cause of the 'bluing' effect could be some gas-phase emission at the K[2.2] band due to circumstellar (or interstellar) molecules (e.g., CO) and/or inadequate dust opacity for the dust shell model. There have been some studies on NIR spectra of AGB stars (e.g., Lançon \& Wood 2000; Le Bertre 2005), but it is not yet clear whether AGB star in our Galaxy and the LMC show similar NIR spectra at the K[2.2] band or not. Though it is not presented in a $2 \mathrm{CD}$ in this paper, we have found that the observed S1[3.6]-S4[8.0] colors for the LMC does not show the 'bluing' effect (see Table 3 ).

The lower panel of Figure 6 shows the WISE-2MASS 2CDs for AGB stars in the SMC. Compared with other IR colors, the observed K[2.2]-W3[12] colors show small per-
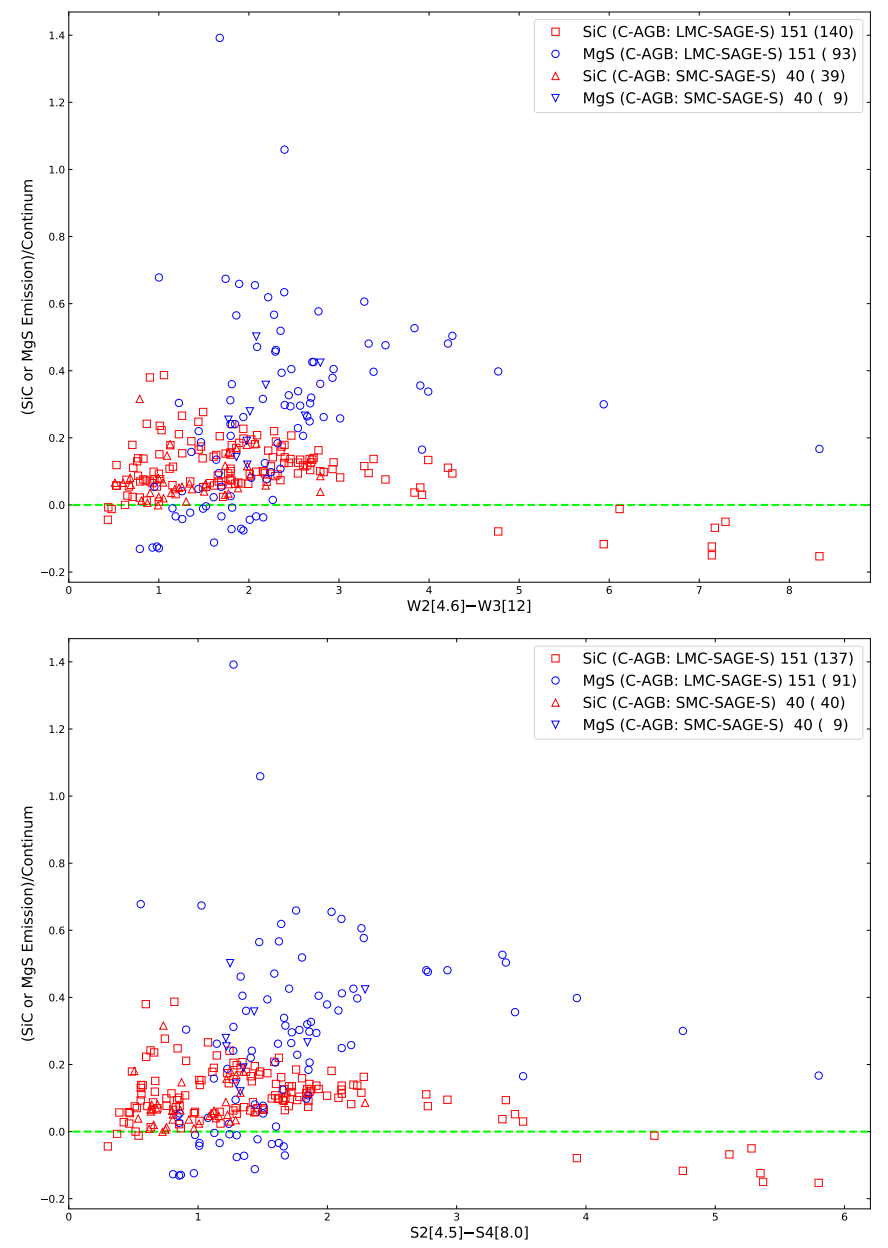

Figure 14. Strength of $\mathrm{SiC}$ and $\mathrm{MgS}$ line emission features in the Spitzer IRS spectra versus IR colors for the C-AGB stars in the (LMC and SMC) SAGE-S samples. The line emission data are from Sloan et al. (2016). For each class, the number of objects is shown. The number in parenthesis denotes the number of the plotted objects with observed data.

centages of the AGB stars with thick dust shell in the SMC (see Table 3). But it is not clear whether the AGB stars in the SMC show the 'bluing' effect or not because other IR colors also show small percentages of the AGB stars with thick dust shells.

\subsection{WISE-Spitzer $2 C D s$}

Figures 7 and 8 show Spitzer-WISE 2CDs. Figure 7 shows the 2CDs using S3[5.8]-S5[24] versus W2[4.6]-W3[12] for AGB stars in our Galaxy and the LMC. Figure 8 shows the Spitzer-WISE 2CDs using W3[12]-S5[24] versus S2[4.5]$\mathrm{S} 4[8.0]$ for AGB stars in our Galaxy and the LMC. The $\mathrm{S} 5$ [24] band can be useful to investigate the $\mathrm{Mg}_{0.9} \mathrm{Fe}_{0.1} \mathrm{~S}$ dust features around $26 \mu \mathrm{m}$ (see Figure 11) for C-AGB stars.

The upper panels of Figures 7 and 8 show the two WISESpitzer 2CDs for our Galaxy, which are less meaningful than other 2CDs because the usable Spitzer data are available only for a minor portion of AGB stars in our Galaxy. For C-AGB 

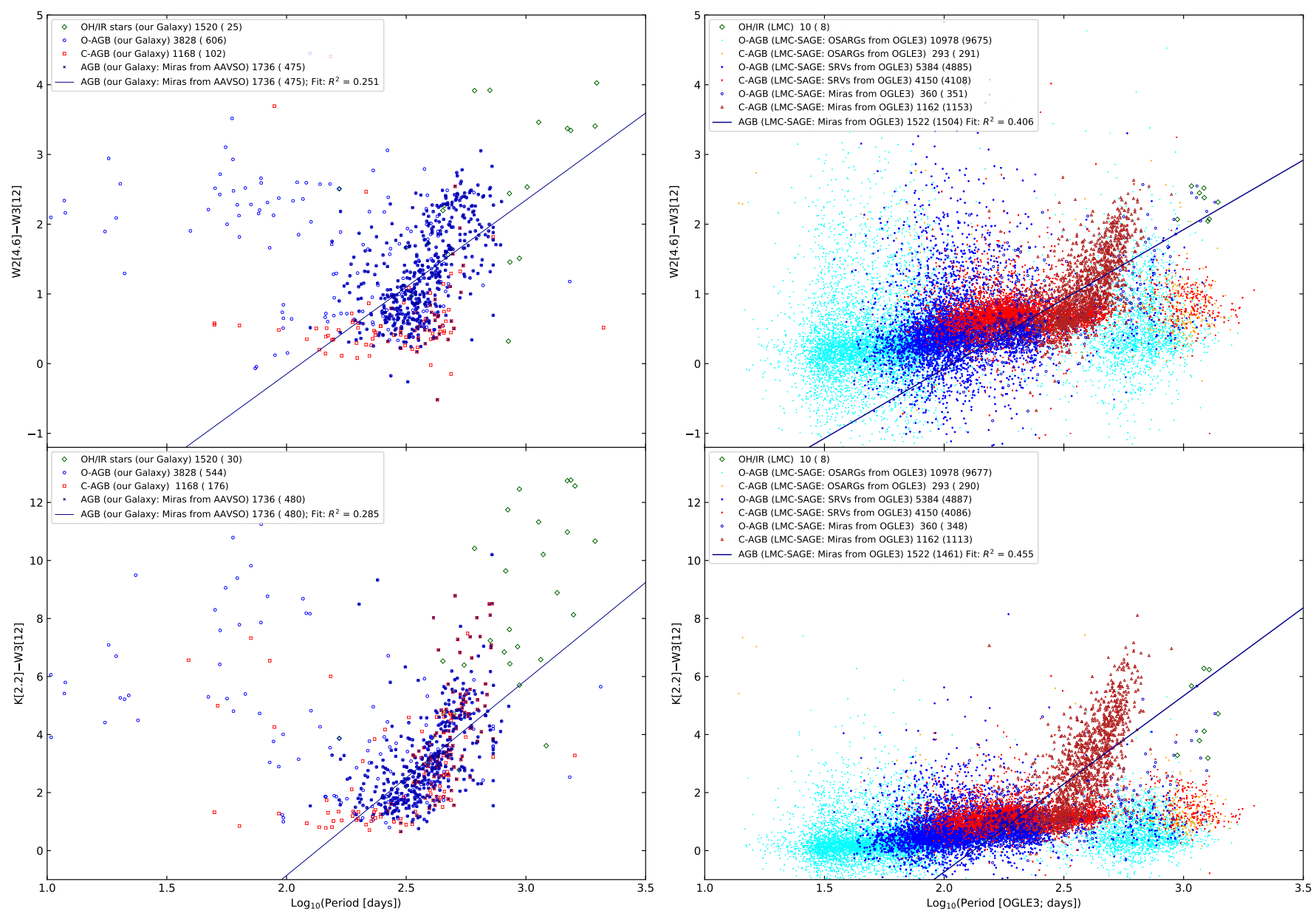

Figure 15. Period-color relations for AGB stars in our Galaxy and the LMC. The periods for AGB stars in our Galaxy are from AAVSO. The periods for OH/IR stars in our Galaxy are from Chen et al. (2001). The periods for OH/IR stars in the LMC are from Goldman et al. (2017). For each class, the number of objects is shown. The number in parenthesis denotes the number of the plotted objects with good quality observed data.

stars, the sample number is too small to find any meanings. Though the sample number is small, the observed data points of Galactic O-AGB stars are located in the wide range $\mathrm{O}$ AGB model colors $\left(\tau_{10}=0.001-40\right)$ on the 2CDs.

The lower panels of Figures 7 and 8 show the two WISESpitzer 2CDs for the LMC. The observations of AGB stars in the LMC show similar properties on both $2 \mathrm{CD}$ s though there are more scatters in the W3[12]-S5[24] color. This could be partly because the W3[12] and S5[24] fluxes are obtained at different pulsation phases.

For O-AGB stars, the ratio of HMOA stars with thick dust shells $\left(\tau_{10}>7\right)$ for the LMC (SAGE-S: 8.1-29.0 \%; SAGE: $0.1-0.2 \%)$ is smaller than the one for our Galaxy (13.8-67.6 $\%$ ) (see Table 3). Observations of C-AGB stars in the LMCSAGE-S sample can be reproduced by wide ranges of the C-AGB dust model colors $\left(\tau_{10}=0.001-5\right)$ using AMC, SiC, and $\mathrm{Mg}_{0.9} \mathrm{Fe}_{0.1} \mathrm{~S}$ dust grains. For C-AGB stars, the effect of the $\mathrm{Mg}_{0.9} \mathrm{Fe}_{0.1} \mathrm{~S}$ and $\mathrm{SiC}$ dust, which shows deviations from the pure AMC model, is more conspicuous on the $2 \mathrm{CD}$ using the W3[12]-S5[24] color (see Figure 8).
Figure 9 shows the two WISE-Spitzer 2CDs for AGB stars in the SMC. For C-AGB stars, the ratio of C-AGB stars with thick dust shells $\left(\tau_{10}>1\right)$ for the SMC (SAGE-S: 2.5-2.7\%; SAGE: $0.1-0.2 \%$ ) is smaller than the one for LMC (SAGE-S: 13.3-13.9\%; SAGE: $0.6 \%$ ) (see Table 3).

\subsection{SiC and $M g S$ features for $C$ - $A G B$ stars}

For C-AGB stars, the SiC dust features at $11.3 \mu \mathrm{m}$ and $\mathrm{Mg}_{0.9} \mathrm{Fe}_{0.1} \mathrm{~S}$ dust features at $28 \mu \mathrm{m}$ can be useful to compare the theoretical models with the observations (see the lower panel of Figure 10). For the $\mathrm{SiC}$ dust feature at $11.3 \mu \mathrm{m}$, the emission feature becomes stronger as $\tau_{10}$ increase up to $\tau_{10}=0.1$, then becomes weaker emission feature, and then it becomes an absorption feature for $\tau_{10}>1$. On IR 2CDs, the observation of C-AGB stars show similar effects.

Figure 14 shows the strength of $\mathrm{SiC}$ and $\mathrm{MgS}$ line emission features in the Spitzer IRS spectra versus IR colors (W2[4.6]-W3[12] and S2[4.5]-S4[8.0]) for the C-AGB stars in the (LMC and SMC) SAGE-S samples. The plots in Figure 14 show the similar effects: the strength become stronger 

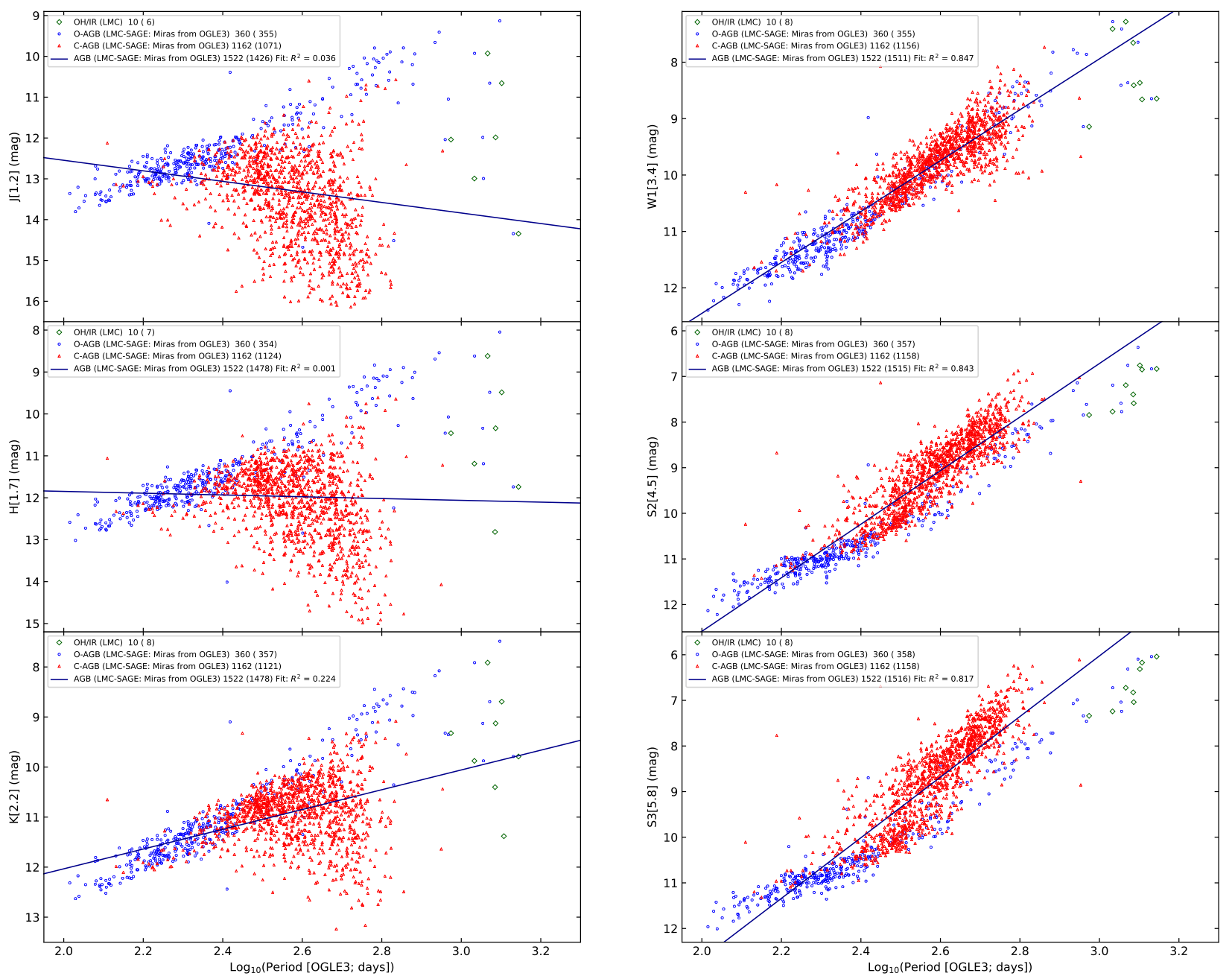

Figure 16. Period-magnitude relations (at NIR and MIR bands) for Mira variables in the LMC-SAGE AGB sample. The periods for OH/IR stars in the LMC are from Goldman et al. (2017). For each class, the number of objects is shown. The number in parenthesis denotes the number of the plotted objects with good quality observed flux data. The coefficient of determination $\left(R^{2}\right)$ of the linear relationship is also shown.

as the color gets redder up to some point, then it becomes weaker.

For C-AGB stars, the theoretical models using AMC dust with a mixture of $\mathrm{SiC}$ and $\mathrm{Mg}_{0.9} \mathrm{Fe}_{0.1} \mathrm{~S}$ grains can reproduce the observations in much wider regions on any IR 2CDs (see Figures 4 - 9).

\section{INFRARED PROPERTIES OF KNOWN PULSATING VARIABLES}

AGB stars are characterized by long-period and large amplitude pulsations. It is generally believed that more evolved (or more massive) AGB stars would have the larger pulsation amplitudes, longer pulsation periods, and higher mass-loss rates (e.g., De Beck et al. 2010; Suh \& Kwon 2013b).

In the sample of 4996 Galactic AGB stars, there are 1736 Miras identified from AAVSO (see Section 2.2). In the sample of 33,537 LMC-SAGE AGB stars, there are 22,327 pulsating variables identified from the OGLE3 (1522 Miras, 9534 SRVs, and 11,271 OSARGs). And in the sample of 5742 SMC-SAGE AGB stars, there are 4837 pulsating vari- ables known from the OGLE3 (341 Miras, 2036 SRVs, and 2460 OSARGs) (see Section 2.4). In this section, we investigate infrared properties of the known pulsating variables in our Galaxy and the Magellanic clouds (SAGE sample).

\subsection{Period-color relations}

Figure 15 shows period-color relations. It shows K[2.2]W3[12] and W2[4.6]-W3[12] colors versus pulsation periods for AGB stars in our Galaxy and the LMC. The left and right panels show the relation for AGB stars in our Galaxy and the LMC, respectively. Though there are large scatters, we find that Mira variables, among all types of variables, show stronger relationship between IR colors and pulsation periods.

During the AGB phase, the more evolved stars with longer pulsation periods would have thicker dust envelopes and redder IR colors. Compared with our Galaxy, we find that Mira variables in the LMC show larger coefficients of determination $\left(R^{2}\right)$ for both IR colors, which mean higher strength of the relationship. This could be because most of the Galactic 

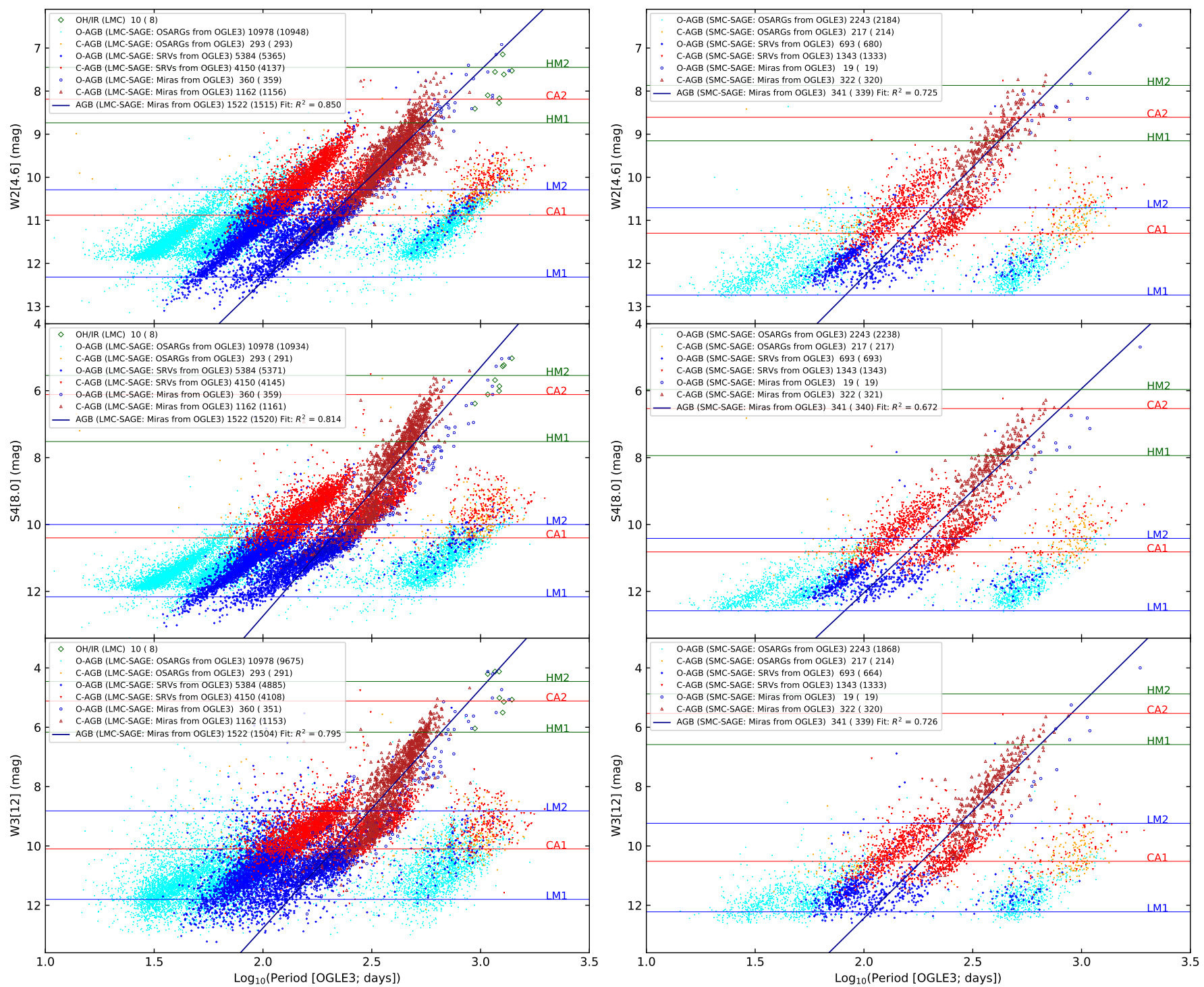

Figure 17. Period-magnitude relations (at NIR and MIR bands) for all AGB stars in the SAGE samples (LMC in the left and SMC in the right panel). The periods for OH/IR stars in the LMC are from Goldman et al. (2017). For each class, the number of objects is shown. The number in parenthesis denotes the number of the plotted objects with good quality observed flux data. The coefficient of determination $\left(R^{2}\right)$ of the linear relationship for Mira variables is shown. The horizontal lines indicate model magnitudes for LMOA (blue), C-AGB (red), and HMOA (green) stars (see Table 5).

AGB stars with thick dust shells are not listed in the AAVSO catalog (see Section 2.2). The AAVSO catalog is mainly based on optical observations, which suffer severe extinctions due to the Galactic disk.

\subsection{Period-magnitude relations}

Compared with AGB stars in our Galaxy, it is easier to study the period-magnitude relation (PMR) for the AGB stars in the Magellanic Clouds because they share similar distances. Among all types of pulsating variables, it is known that Mira variables show a stronger relationship between the IR fluxes and pulsation periods (e.g., Soszyński et al. 2009).

Figure 16 shows the relation for Miras in the LMC-SAGE sample at NIR and MIR bands. Though the relation shows very large scatters at the 2MASS bands (J[1.2], H[1.7], and $\mathrm{K}[2.2]$ ) and shorter wavelengths, the Miras show a strong linear relationship when the wavelength is longer than about 3 $\mu \mathrm{m}$ (W1[3.4], S2[4.5], and S3[5.8]). We find that Mira variables in the LMC show fairly large coefficients of determination $\left(R^{2}=0.6-0.85\right)$ of the linear relationship at the wavelength bands in the range $3-24 \mu \mathrm{m}$.

Figure 17 shows the PMRs at NIR and MIR bands (W2[4.6], S4[8.0], and W3[12]) for SAGE AGB sample stars in the Magellanic Clouds that are identified as pulsating variables from OGLE3. The plots show the objects of different variable types and chemical classifications. Again, we find that the Mira variables in the LMC and SMC show fairly strong linear relationships. 

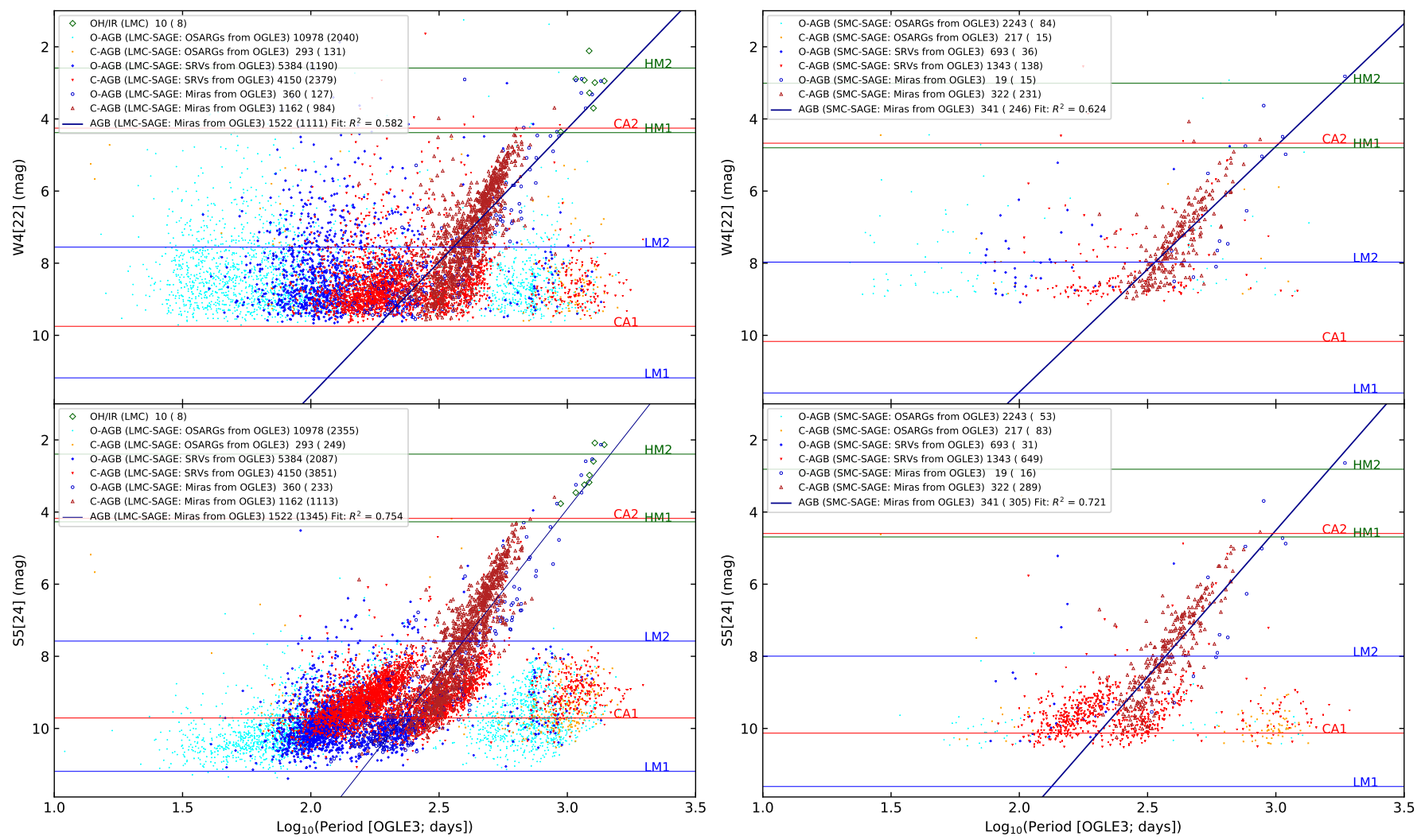

Figure 18. Period-magnitude relations (at MIR bands) for all AGB stars in the SAGE samples (LMC in the left and SMC in the right panel). The periods for OH/IR stars in the LMC are from Goldman et al. (2017). For each class, the number of objects is shown. The number in parenthesis denotes the number of the plotted objects with good quality observed flux data. The coefficient of determination $\left(R^{2}\right)$ of the linear relationship for Mira variables is shown. The horizontal lines indicate model magnitudes for LMOA (blue), C-AGB (red), and HMOA (green) stars (see Table 5).

Figure 18 shows the PMRs at longer wavelength bands (W4[22] and S5[24]). In the two plots, low brightness OAGB stars are relatively deficient compared with the three plots at shorter wavelengths (see Figure 15). This would be because of the lower sensitivity of the detectors (W4[22]: 5.4 mJy, S5[24]: $0.11 \mathrm{mJy}$; see Section 2.1). Because of the even lower sensitivity, the W4[22] band cannot detect more low brightness objects (dimmer than about $9.5 \mathrm{mag}$ ). Therefore, the upper panel is more deficient in low brightness O-AGB stars (mostly OSARGs). We expect that there would be as many low brightness O-AGB stars as those in Figure 18 if we had detectors with higher sensitivities.

\section{MAGNITUDE DISTRIBUTIONS AT MIR BANDS FOR AGB STARS IN THE MAGELLANIC CLOUDS}

Magnitude distributions at MIR bands for different classes of AGB stars can be useful to study the nature of the galaxy. Compared with Galactic AGB stars, it is much easier to investigate the magnitude distributions for the AGB stars in the Magellanic Clouds because they share similar distances and they are relatively freer from interstellar extinctions. In Section 6.2, we presented PMRs at IR bands for known pulsating variables. In this section, we present the brightness distributions at MIR bands for all AGB stars in the SAGE samples

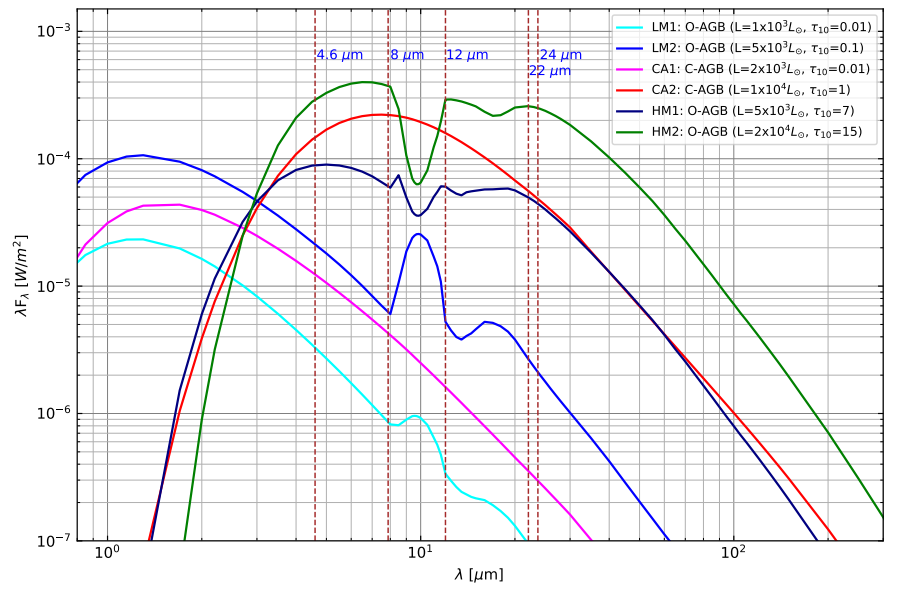

Figure 19. Model SEDs of the six model for LMOA, C-AGB, and HMOA stars (see Table 5) using the distance of $1 \mathrm{pc}$.

of the LMC and SMC and compare them with the theoretical model magnitudes for typical AGB stars.

\subsection{Theoretical model magnitudes}

We may obtain the theoretical model magnitude at given wavelength band from the model SED using the ZMF at the 

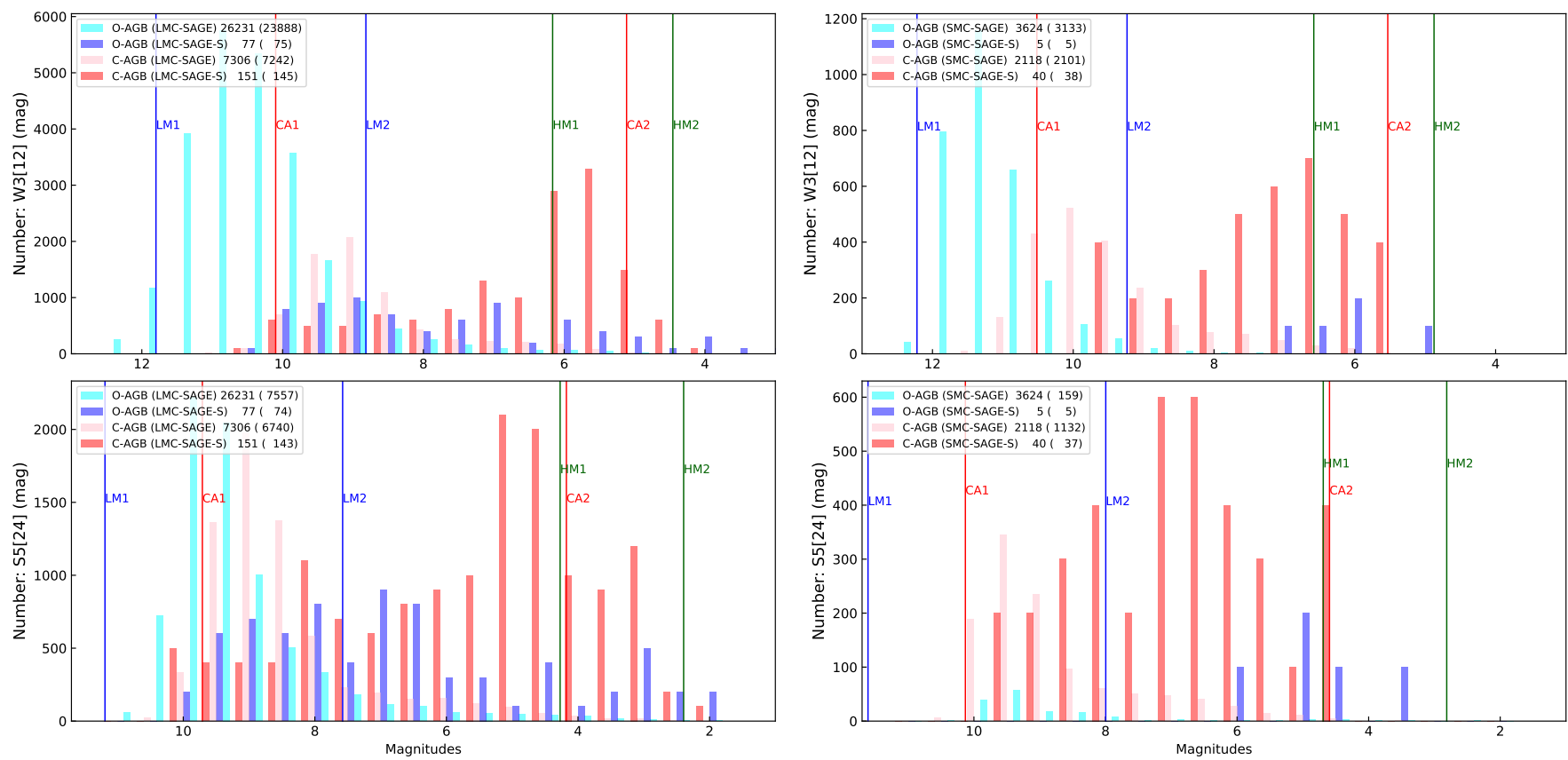

Figure 20. Magnitude distributions at MIR bands for all AGB stars in the SAGE samples (LMC in the left and SMC in the right panel). For each class, the number of objects is shown. The number in parenthesis denotes the number of the observed objects. The numbers of the observed objects in the SAGE-S samples have been multiplied by 100 for the plots. The vertical lines indicate model magnitudes for LMOA (blue), C-AGB (red), and HMOA (green) stars (see Table 5).

wavelength band (see Table 2). Because the shape of the model SED is independent on the luminosity of the central star when all other parameters are fixed, the DUSTY code (Ivezić \& Elitzur 1997) calculates the model SED only in relative scale (see Section 4.1). To calculate the model SED in absolute scale for given luminosity and distance, we use the RADMC-3D code http://www.ita.uni-heidelberg. $\mathrm{de} / \sim$ dullemond/software/radmc-3d/) in conjunction with the DUSTY code. The RADMC-3D models using the same scheme as used by Suh \& Kwon (2013a) and the same model parameters as the DUSTY model produce almost identical results.

We obtain the model magnitudes from the model SED in absolute scales for given luminosity of the central star and distance. For the RADMC-3D models, we use the same model parameters as the DUSTY model used in this work (see Section 4.1) except for the luminosity of the central star. Table 5 lists the parameters for six models of typical AGB star with various luminosity of the central star. Figure 19 shows the model SEDs for the six models for LMOA stars, C-AGB stars, and HMOA stars in absolute scales when we use the distance of $1 \mathrm{pc}$.

In Figures 17 and 18, the horizontal lines indicate the model magnitudes for the six different models for LMOA, C-AGB, and HMOA stars (see Table 5 and Figure 19). We assume that the distances of the LMC and SMC are 49.97 and $60.6 \mathrm{kpc}$, respectively.

\subsection{Magnitude distributions at MIR bands}

Table 5. Six models for typical AGB stars

\begin{tabular}{llllll}
\hline \hline Model & Class & Dust $^{1}$ & $\tau_{10}$ & $T_{*}(\mathrm{~K})$ & $L_{*}\left(10^{3} L_{\odot}\right)$ \\
\hline LM1 & LMOA & silicate & 0.01 & 3000 & 1 \\
LM2 & LMOA & silicate & 0.1 & 2500 & 5 \\
CA1 & C-AGB & AMC & 0.1 & 2500 & 2 \\
CA2 & C-AGB & AMC & 1 & 2000 & 10 \\
HM1 & HMOA & silicate & 7 & 2000 & 5 \\
HM2 & HMOA & silicate & 15 & 2000 & 20 \\
\hline
\end{tabular}

${ }^{1}$ See Section 4.1 for details. For all models, $T_{c}=1000 \mathrm{~K}$.

Figure 20 shows magnitude distributions at two MIR bands (W3[12] and S5[24]) for all AGB stars in the SAGE samples (LMC and SMC). We choose the two MIR bands (W3[12] and S5[24]) because they can be good measures of the luminosity for the objects with thick dust shells. The vertical lines indicate the model magnitudes for the six different models for LMOA, C-AGB, and HMOA stars (see Table 5).

Table 6 lists the percentages of bright stars at MIR bands. The weighted averaged percentages of bright O-AGB stars (brighter than model HM2) in the LMC and SMC are 1.76 $\%$ and $0.07 \%$, respectively. And the weighted averaged percentages of bright C-AGB stars (brighter than model CA2) in the LMC and SMC are $8.33 \%$ and $0.97 \%$, respectively.

Based on the magnitudes at wavelength bands in the range 4-24 $\mu \mathrm{m}$ (see Figures $17-20$ ), we may roughly divide the 
Table 6. Percentages ${ }^{1}$ of bright AGB stars at MIR bands. See Figure 20 .

\begin{tabular}{lllll}
\hline \hline Class & W3[12] & S5[24] & Average & WA $^{2}$ \\
\hline C-AGB (LMC-SAGE) & $0.29(7242)$ & $0.65(6740)$ & 0.46 & 8.33 \\
C-AGB (LMC-SAGE-S) & $6.21(145)$ & $18.18(143)$ & 12.15 & - \\
C-AGB (SMC-SAGE) & $0.10(2101)$ & $0.18(1132)$ & 0.12 & 0.97 \\
C-AGB (SMC-SAGE-S) & $0.00(38)$ & $2.70(37)$ & 1.33 & - \\
\hline O-AGB (LMC-SAGE) & $0.042(23888)$ & $0.066(7557)$ & 0.048 & 1.76 \\
O-AGB (LMC-SAGE-S) & $6.67(75)$ & $4.05(74)$ & 5.37 & - \\
O-AGB (SMC-SAGE) & $0.064(3133)$ & $0.63(159)$ & 0.091 & 0.07 \\
O-AGB (SMC-SAGE-S) & $0.00(5)$ & $0(5)$ & 0.00 & - \\
\hline
\end{tabular}

${ }^{1}$ The percentages of the C-AGB (O-AGB) stars that are brighter than model CA2 (HM2) (see Table 5). ${ }^{2}$ The weighted averaged percentages for the LMC and SMC, which assumes that the numbers of observed objects in the SAGE-S samples are multiplied by 100 . The number in parenthesis denotes the number of the observed objects plotted in Figure 20.

AGB stars in the LMC and SMC into three groups: (1) a large group of low brightness O-AGB stars (LMOA stars) including most of the OSARGs, (2) a large group of intermediate brightness C-AGB stars, and (3) a small group of bright O-AGB stars (HMOA stars) including OH/IR stars. See also Table 7.

We find that the LMC is deficient in the O-AGB stars that are bright at MIR bands and the SMC is more deficient in those stars. Though it can not be confirmed in this work because it requires distance information for the large sample of Galactic AGB star, we expect that there would be abundant O-AGB stars that are bright at MIR bands in our Galaxy. 1520 OH/IR stars are identified in our Galaxy (see Section 2.5), from which many of them are known to be as bright as $3 \times 10^{4} L_{\odot}$ at maximum phases (e.g., Suh 2004; Suh \& Kwon 2013a). We expect that there would be more bright O-AGB stars including many extreme OH/IR stars in the third group if we could make similar plots (see Figures 17 - 20) for AGB stars in our Galaxy.

\section{DISCUSSION: AGB STARS IN OUR GALAXY AND THE MAGELLANIC CLOUDS}

Compared with our Galaxy, we find that the LMC and SMC are deficient in O-AGB stars with thick dust shells (or large dust optical depths) on any IR 2CDs (see Section 5). The weighted averaged percentages of HMOA stars with thick dust shells $\left(\tau_{10}>7\right)$ for our Galaxy $(17.2 \%)$ is larger than the ones for the LMC (3.4\%) and SMC (1.8\%) SAGE sample stars (see Table 4). This could be because the highmass star formation is less active in the Magellanic Clouds than in our Galaxy. O-AGB stars with thick dust shells or $\mathrm{OH} / \mathrm{IR}$ stars are generally considered to be more massive OAGB stars. Up to now, $1520 \mathrm{OH} / \mathrm{IR}$ stars are identified in our Galaxy, $10 \mathrm{OH} / \mathrm{IR}$ stars are identified in the LMC, and no OH/IR star in the SMC is identified yet (see Section 2.5).

Compared with our Galaxy, we find that the LMC and SMC are deficient in C-AGB stars with thick dust shells on the IR 2CDs (see Section 5). The weighted averaged percentages of C-AGB stars with thick dust shells $\left(\tau_{10}>1\right)$ for our
Galaxy $(17.1 \%)$ is larger than the ones for the LMC $(9.4 \%)$ and SMC (1.3\%) SAGE sample stars (see Table 4). This is in accord with Ventura et al. (2016) who studied infrared properties of C-AGB stars in the Magellanic Clouds found that the infrared colors of $\mathrm{C}$-AGB stars in the LMC are redder compared to their counterparts in the SMC.

It is believed the stars with initial masses in the intermediate mass range can become C-AGB stars (see Section 1). Compared with the LMC, the SMC is more deficient in CAGB stars with thick dust shells. Also Nanni et al. (2019) found a group of C-AGB stars with high mass-loss rates in the LMC that is not present in the SMC. This could be because the initial masses of C-AGB stars in the LMC are larger than those in the SMC (Ventura et al. 2016).

In the study of the magnitude distributions at MIR bands for AGB stars in the Magellanic Clouds, we find that the LMC is deficient in AGB stars that are bright at MIR bands and the SMC is more deficient in those stars (see Section 7.2 and Table 6). Brighter AGB stars at MIR bands are generally considered to be more massive AGB stars with thick dust shells.

Compared with our Galaxy, the LMC looks to be deficient in O-AGB and C-AGB stars that are bright at MIR bands and have thick dust shells. And the SMC looks to be more deficient in those stars. This could be because Magellanic Clouds are more metal poor than our Galaxy and the LMC is more metal rich than the SMC. It is known that the metallicity, gaseous content, and historical star formation rate of the LMC lies midway between those of our Galaxy and the SMC. The LMC's metallicity is about $50 \%$ that of the Sun while the SMC's is only about $20 \%$ (e.g., Madden et al. 2013; Höfner \& Olofsson 2018). In a galaxy with a higher metallicty (and a lower gas-to-dust ratio), the star formation in a higher mass range would be more active and the galaxy would have a higher ratio of AGB stars with thick dust shells, which are bright at MIR bands.

Table 7 summarizes overall distributions of AGB classes in our Galaxy and the Magellanic Clouds based on our studies of the IR 2CDs (see Section 5) and magnitudes distributions at MIR bands (see Section 7).

\section{SUMMARY}

We have investigated infrared properties of AGB stars in our Galaxy and the Magellanic Clouds using various infrared observational data and theoretical models. We have used catalogs for the sample of 4996 AGB stars in our Galaxy and about 39,000 AGB stars in the Magellanic Clouds from the available literature.

For each object in the sample, we have cross-identified the 2MASS, WISE, and Spitzer counterparts. To compare the physical properties of O-AGB and C-AGB stars in our Galaxy and the Magellanic Clouds, we have presented IR 2CDs by using the 2MASS, WISE, and Spitzer photometric data.

The IR 2CDs are useful to compare IR properties of AGB stars in our Galaxy and the Magellanic Clouds with theoretical models. For AGB stars in our Galaxy, the IR 2CDs using 
Table 7. Overall distributions of AGB classes based on the IR 2CDs and IR magnitudes

\begin{tabular}{|c|c|c|c|c|c|}
\hline $\begin{array}{l}\text { Class } \\
\left(\text { Mass }^{1}\right)\end{array}$ & $\begin{array}{l}\text { Luminosity } \\
\left(10^{4} L_{\odot}\right)\end{array}$ & $\begin{array}{l}\text { Dust } \\
\left(\tau_{10}\right)\end{array}$ & Our Galaxy & LMC & SMC \\
\hline $\begin{array}{l}\text { LMOA } \\
(0.5-1.55)\end{array}$ & $\begin{array}{l}\text { less luminous } \\
(0.1-0.5)\end{array}$ & $\begin{array}{l}\text { silicate } \\
(0.001-3)\end{array}$ & $\mathrm{AB}$ & $\mathrm{AB}$ & $\mathrm{AB}$ \\
\hline LMOA:C ${ }^{2}$ & - & $<0.1$ & ${ }^{4} 27.1 \%$ & $79.5 \%$ & $82.7 \%$ \\
\hline LMOA:M ${ }^{3}$ & $\mathrm{~L}<\mathrm{LM} 2$ & - & $\mathrm{AB}^{5}$ & ${ }^{6} 83.0 \%$ & ${ }^{6} 85.0 \%$ \\
\hline $\begin{array}{l}\text { C-AGB } \\
(1.55-4)\end{array}$ & $\begin{array}{l}\text { luminous } \\
(0.2-1)\end{array}$ & $\begin{array}{l}\text { AMC } \\
(0.001-5)\end{array}$ & $\mathrm{AB}$ & DH & $\mathrm{MDH}$ \\
\hline C-AGB: $\mathrm{C}^{2}$ & - & $>1$ & $17.1 \%$ & $9.4 \%$ & $1.3 \%$ \\
\hline C-AGB:M ${ }^{3}$ & $\mathrm{~L}>\mathrm{CA} 2$ & - & $\mathrm{AB}^{5}$ & $8.33 \%$ & $0.97 \%$ \\
\hline $\begin{array}{l}\text { HMOA } \\
(4-10)\end{array}$ & $\begin{array}{l}\text { more luminous } \\
(0.5-3)\end{array}$ & $\begin{array}{l}\text { silicate } \\
(3-40)\end{array}$ & $\mathrm{AB}$ & $\mathrm{DH}$ & MDH \\
\hline HMOA:C ${ }^{2}$ & - & $>7$ & $17.2 \%$ & $3.4 \%$ & $1.8 \%$ \\
\hline HMOA:C ${ }^{2}$ & - & $>15$ & $7.0 \%$ & $0.44 \%$ & $0.048 \%$ \\
\hline HMOA: $\mathrm{M}^{3}$ & $\mathrm{~L}>\mathrm{HM} 2$ & - & $\mathrm{AB}^{5}$ & $1.76 \%$ & $0.07 \%$ \\
\hline
\end{tabular}

Acronyms; AB: abundant, DH: deficient in high-mass stars, MDH: more deficient in high-mass stars. ${ }^{1}$ the mass range in $M_{\odot}$ (see Section 1). ${ }^{2}$ Based on the IR 2CDs (see Section 5 and Table 4). ${ }^{3}$ Based on the magnitude distributions at MIR bands (see Section 7 and Table 6). ${ }^{4}$ This could be due to a selection effect (see Section 5.1). ${ }^{5}$ This can not be confirmed in this work because it requires distance information for the large sample of the Galactic AGB stars (see Section 7.2). ${ }^{6}$ Obtained from Figure 20 at the MIR band W3[12] because the S5[24] band would not detect all of the $\operatorname{dim}$ AGB stars with thin dust shells (see Section 6.2).

Spitzer photometric data are less useful because the reliable data are available only for a small number of objects.

We have performed radiative transfer model calculations for AGB stars using various possible parameters of central stars and spherically symmetric dust shells. We have compared the various theoretical models with the observations of AGB stars on the IR 2CDs.

All AGBs stars in our Galaxy and the Magellanic Clouds look similar in dust properties. The theoretical dust shell models can roughly explain the observations of AGB stars in our Galaxy and the Magellanic Clouds on various IR 2CDs using dust opacity functions of amorphous silicate and amorphous carbon. For LMOA stars, the silicate dust with a mixture of amorphous alumina $\left(\mathrm{Al}_{2} \mathrm{O}_{3}\right)$ and $\mathrm{Fe}-\mathrm{Mg}$ oxides can explain wider regions on the IR 2CDs. For C-AGB stars, AMC dust with a mixture of $\mathrm{SiC}$ and $\mathrm{Mg}_{0.9} \mathrm{Fe}_{0.1} \mathrm{~S}$ grains can reproduce the observations in much wider regions on the 2CDs.
The observed K[2.2]-W3[12] colors for AGB stars are bluer than the theoretical dust shell models, which do not consider gas-phase radiation processes. This 'bluing' effect looks to be stronger for AGB stars in the LMC than for those in our Galaxy. The 'bluing' effect could be due to circumstellar (or interstellar) molecules and/or inadequate dust opacity, but further investigations on the NIR spectra of AGB stars are necessary to clarify it.

Compared with our Galaxy, we have found that the Magellanic Clouds are deficient in O-AGB and C-AGB stars with thick dust shells on the IR 2CDs. AGB stars with thick dust shells are generally considered to be more massive (or more evolved). Compared with the LMC, the SMC is more deficient in the AGB stars with thick dust shells. This could be because the high-mass star formation is less active in the Magellanic Clouds than in our Galaxy. It is known that the Magellanic Clouds are more metal poor than our Galaxy and the LMC is more metal rich than the SMC.

We have investigated period-magnitude relations for known pulsating variables in the LMC and SMC. The Mira variables show a strong linear relationship at the wavelength bands in the range $3-24 \mu \mathrm{m}$.

We have investigated the magnitude distributions at MIR bands for AGB stars in the LMC and SMC. The LMC and SMC look to be deficient in bright O-AGB and C-AGB stars at MIR bands. Compared with the LMC, the SMC is more deficient in the bright AGB stars. Again, this could be because they are more metal poor and less active in high-mass star formation compared with our Galaxy.

\section{ACKNOWLEDGMENTS}

I thank the anonymous referee for constructive comments and suggestions. This work was supported by the National Research Foundation of Korea (NRF) grant funded by the Korea government (MSIT; Ministry of Science and ICT) (No. NRF-2017R1A2B4002328). This research has made use of the VizieR catalogue access tool, CDS, Strasbourg, France. This research has made use of the NASA/ IPAC Infrared Science Archive, which is operated by the Jet Propulsion Laboratory, California Institute of Technology, under contract with the National Aeronautics and Space Administration.

\section{REFERENCES}

Begemann, B., Dorschner, J., Henning, T., Mutschke, H., \&

Thamm, E. 1994, ApJ, 423, L71

Begemann, B., Dorschner, J., Henning, T., et al. 1997, ApJ, 476, 199

Beichman, C. A., Neugebauer, G., Habing H., Clegg, P. E., \&

Chester, T. C. 1988, IRAS Catalogs and Atlases: Explanatory

Supplement, NASA RP-1190 (Washington: NASA)

Blöcker, T., Herwig, F., \& Driebe, T. 2000, MmSAI, 71, 711
Boyer, M. L., Srinivasan, S., van Loon, J. T., et al. 2011, AJ, 142, 103

Chen, P. S., Szczerba, R., Kwok, S., \& Volk, K. 2001, A\&A, 368, 1006

Cohen, M., Wheaton, W. A., \& Megeath, S. T. 2003, AJ, 126, 1090

Cutri, R. M., Skrutskie, M. F., Van Dyk, S., et al. 2003, The IRSA

2MASS All-Sky Point Source Catalog, NASA/IPAC Infrared

Science Archive

De Beck, E., Decin, L., de Koter, A., et al. 2010, A\&A, 523, A18 
Draine, B. T., Dale, D. A., Bendo, G., et al. 2007, ApJ, 663, 866

Gehrz, R. D., Roellig, T. L., Werner, M. W., et al. 2007, RScI, 78, 011302

Goldman, S. R., van Loon, J. Th., Zijlstra, A. A., et al. 2017, MNRAS, 465, 403

Goldman, Steven R., van Loon, J. Th., Gómez, J. F., et al. 2018, MNRAS, 473, 3835

Gonneau, A., Lancon, A., Trager, S. C., et al. 2016, A\&A, 589, A36

González-Lópezlira, R. A. 2018, ApJ, 856, 170

Groenewegen, M. A. T., van den Hoek, L. B., \& de Jong, T. 1995, A\&A, 293, 381

Groenewegen, M. A. T., \& Sloan, G. C. 2018, A\&A, 609, A114

Henning, T., Begemann, B., Mutschke, H., \& Dorschner, J. 1995, A\&AS, 112, 143

Höfner, S., \& Olofsson, H., 2018, A\&A Rev., 26, 1

Hony, S., Waters, L. B. F. M., \& Tielens, A. G. G. M. 2002, A\&A, 390,533

Iben, I., \& Renzini, A. 1983, ARA\&A, 21, 271

Ivezić, A., \& Elitzur, M. 1997, MNRAS, 287, 799

Jarrett, T. H., Cohen, M., Masci, F., et al. 2011, ApJ, 735, 112

Jones, O. C., Kemper, F., Srinivasan, S., et al. 2014, MNRAS, 440, 631

Jones, O. C., Woods, P. M., Kemper, F. et al. 2017, MNRAS, 470, 3250

Kraemer, K. E.; Sloan, G. C.; Wood, P. R.; Jones, O. C.; Egan, M. P, 2017, ApJ,.834,.185

Kwon, Y.-J., \& Suh, K.-W. 2012, JKAS, 45, 139

Lançon, A., \& Wood, P. R. 2000, A\&AS, 146, 217

Le Bertre, T., Tanaka, M., Yamamura, I., Murakami, H., \& MacConnell, D. J. 2005, PASP, 117, 199

Loup, C., Forveille, T., Omont, A., \& Paul, J. F. 1993, A\&AS, 99, 291

Madden, S. C., Rémy-Ruyer, A., Galametz, M., et al. 2013, PASP, 125,600
Meixner, M., Gordon, K. D., Indebetouw, R., et al. 2006, AJ, 132, 2268

Nanni, A., Groenewegen, M. A. T., Aringer, B., et al. 2019, MNRAS, 487, 502

Pégourié, B. 1988, A\&A, 194, 335

Riebel, D., Srinivasan, S., Sargent, B.,\& Meixner, M. 2012, ApJ, 753, 71

Siess, L. 2006, A\&A, 448, 717

Sloan, G. C., Kraemer, K. E., McDonald, I., et al. 2016, ApJ, 826, 44

Soszyński, I., Udalski, A., Szymański, M. K., et al. 2009, AcA, 59, 239

Soszyński, I., Udalski, A., Szymański, M. K., et al. 2011, AcA, 61, 217

Srinivasan, S., Boyer, M. L., Kemper, F., et al. 2016, MNRAS, 457, 2814

Suh, K.-W. 1999, MNRAS, 304, 389

Suh, K.-W. 2000, MNRAS, 315, 740

Suh, K.-W. 2002, MNRAS, 332, 513

Suh, K.-W. 2004, ApJ, 615, 485

Suh, K.-W. 2014, JKAS, 47, 219

Suh, K.-W. 2015, ApJ, 808, 165

Suh, K.-W. 2016, JKAS, 49, 127

Suh, K.-W. 2018, JKAS, 51, 155

Suh, K.-W., \& Kwon, Y.-J. 2011, MNRAS, 417, 3047

Suh, K.-W., \& Kwon, Y.-J. 2013a, ApJ, 762, 113

Suh, K.-W., \& Kwon, Y.-J. 2013b, JKAS, 46, 235

Suh, K.-W., \& Hong, J. 2017, JKAS, 50, 131

Th. Posch, F., Kerschbaum, H., Mutschke, J., et al. 2002, A\&A, 393, L7

Ventura, P., Karakas, A. I., Dell'Agli, F., et al. 2016, MNRAS, 457, 1456

Watson, C., Henden, A. A., \& Price, A. 2019, yCat, 102027W, 0

Wright, E. L., Eisenhardt, P. R. M., Mainzer, A. K., et al. 2010, AJ, 140,1868 